\title{
Emission factors and chemical characterisation of fine particulate emissions from modern and old residential biomass heating systems determined for typical load cycles
}

\section{Emissionsfaktoren und chemische Charakterisierung von Feinstaubemissionen moderner und alter Biomasse-Kleinfeuerungen über typische Tageslastverläufe}

Joachim Kelz', Thomas Brunner ${ }^{* 1,2,3}$, Ingwald Obernberger ${ }^{1,2,3}$

\begin{abstract}
It is already well known that there are significant differences regarding the emissions, especially particulate matter (PM) emissions, of old and modern as well as automatically and not automatically controlled biomass based residential heating systems. This concerns their magnitude as well as their chemical composition. In order to investigate emission factors for particulate emissions and the chemical compositions of the PM emissions over typical whole day operation cycles, a project on the determination and characterisation of PM emissions from the most relevant small-scale biomass combustion systems was performed at the BIOENERGY 2020+ GmbH, Graz, Austria, in cooperation with the Institute for Process and Particle Engineering, Graz University of Technology. The project was based on test stand measurements, during which relevant operation parameters (gaseous emissions, boiler load, flue gas temperature, combustion chamber temperature etc.) as well as PM emissions have been measured and PM samples have been taken and forwarded to chemical analyses. Firstly, typical whole day operation cycles for residential biomass combustion systems were specified for the test runs. Thereby automatically fed and automatically controlled boilers, manually fed and automatically controlled boilers as well as manually fed stoves were distinguished. The results show a clear correlation between the gaseous emissions (CO and $\mathrm{OGC}$ ) and the PM, emissions. It is indicated that modern biomass combustion systems emit significantly less gaseous and PM emissions than older technologies (up to a factor of 100). Moreover, automatically fed systems emit much less gaseous and PM emissions than manually fed batch-combustion systems. PM emissions from modern and automatically controlled systems mainly consist of alkaline metal salts, while organic aerosols and soot dominate the composition of aerosols from old and not automatically controlled systems. As an important result comprehensive data concerning gaseous and PM emissions of different old and modern biomass combustion systems over whole day operation cycles are now available. Derived from these data, correlations between burnout quality, particulate emissions as well as particle composition of the PM emissions can be deduced.
\end{abstract}

Keywords aerosols, small-scale biomass combustion systems, whole day operation cycle, chemical composition

*Correspondence: Thomas.brunner@bioenergy2020.eu

'BIOENERGY 2020+ GmbH, Inffeldgasse 21b, A-8010 Graz, Österreich

Full list of author information is available at the end of the article 


\begin{abstract}
Kurzfassung
Es ist allgemein bekannt, dass es signifikante Unterschiede bezüglich partikelförmiger Emissionen von modernen und alten sowie automatisch und nicht automatisch geregelten Biomasse-Kleinfeuerungsanlagen gibt. Die Unterschiede beziehen sich einerseits auf die Höhe der Partikelemission und andererseits auf deren chemische Zusammensetzung. Um Emissionsfaktoren für partikelförmige Emissionen über typische realitätsnahe Tageslastverläufe zu bestimmen und die Feinstaubemissionen chemisch zu charakterisieren, wurde ein Projekt an der BIOENERGY 2020+ GmbH, Graz, Österreich, in Kooperation mit dem Institut für Prozess- und Partikeltechnik, Technische Universität Graz, Österreich, initiiert. Fünf moderne, dem aktuellen Stand der Technik entsprechende, Biomasse-Kleinfeuerungen sowie zwei Altanlagen, die den aktuellen Anlagenbestand widerspiegeln, wurden im Zuge von Testläufen an einem Teststand untersucht. Dabei wurden alle relevanten Betriebsparameter der Biomasse-Kleinfeuerungen sowie die im Zuge des Verbrennungsprozesses entstehenden gas- und partikelförmigen Emissionen kontinuierlich gemessen und aufgezeichnet. Des Weiteren wurden Partikelemissionsproben gezogen und anschließend chemisch analysiert. Für die Testläufe wurden typische Tageslastverläufe, die den Betrieb dieser Feuerungen in der Praxis abbilden, berücksichtigt. Dabei wurde zwischen automatisch beschickten und automatisch geregelten Kesseln, manuell beschickten und automatisch geregelten Kesseln sowie manuell beschickten Naturzugöfen unterschieden. Die Ergebnisse zeigen einen deutlichen Zusammenhang zwischen den gasförmigen Emissionen, die durch unvollständigen Gasphasenausbrand (CO- und org.C-Emissionen) entstehen, und den Feinstaubemissionen. Es wurde festgestellt, dass moderne Biomasse-Kleinfeuerungsanlagen wesentlich (bis zu einem Faktor 100) geringere gas- und partikelförmige Emissionen aufweisen als ältere Technologien. Weiters emittieren automatisch beschickte Systeme signifikant geringere gas- und partikelförmige Emissionen als händisch beschickte Systeme. Die Feinstaubemissionen von modernen und automatisch geregelten Biomasse-Kleinfeuerungen bestehen zum Großteil aus Alkalimetallverbindungen, während organische Kohlenstoffverbindungen und Russ die Zusammensetzung der Feinstäube aus alten und nicht geregelten Systemen dominieren. Es stehen nun erstmals umfangreiche Daten zu gas- und partikelförmigen Emissionen über für die jeweilige Biomasse-Kleinfeuerung typische, realitätsnahe Tageslastverläufe zur Verfügung, die deutliche Korrelationen zwischen Ausbrandqualität, Partikelemission und chemischer Zusammensetzung der Partikel zeigen.
\end{abstract}

Schlüsselwörter Feinstaub, Biomasse-Kleinfeuerungen, Tageslastverlauf, chemische Zusammensetzung

\section{Inhalte und Zielsetzungen}

Aufgrund steigender Bemühungen die $\mathrm{CO}_{2}$-Emissionen $\mathrm{zu}$ reduzieren, hat die energetische Verwertung von Biomasse in den letzten Jahren wesentlich an Bedeutung gewonnen. Die Verbrennung von chemisch unbehandeltem Holz in Kleinfeuerungsanlagen leistet europaweit einen erheblichen Beitrag zur Bereitstellung von Raumwärme und Warmwasser. Aktuell werden verschiedenste Biomasse-Kleinfeuerungstechnologien wie Pellet-, Hackgutund Stückholzkessel sowie unterschiedliche Naturzugöfen (Kaminöfen und Kachelöfen) eingesetzt, wobei Stückholzkessel und mit Stückholz befeuerte Naturzugöfen den überwiegenden Anteil der derzeit installierten Systeme darstellen. Durch den gegenüber modernen, dem aktuellen Stand der Technik entsprechenden, Systemen geringeren technologischen Standard, emittieren ältere Biomasse-Kleinfeuerungen wesentlich mehr gas- und partikelförmige Emissionen. Dies führt wiederum dazu, dass die Verbrennung von Biomasse in Kleinfeuerungsanlagen in vielen europäischen Regionen einer der Hauptverursacher von Feinstaubemissionen ist.

Umfassende wissenschaftliche Studien [1-7] haben gezeigt, dass es signifikante Unterschiede bezüglich der Emissionen, speziell der Partikelemissionen, von modernen und alten sowie automatisch und nicht automatisch geregelten Biomasse-Kleinfeuerungsanlagen gibt, sowie dass in erster Linie kohlenstoffhaltige Partikel toxikologisch relevant zu sein scheinen [8]. Die Unterschiede beziehen sich einerseits auf die Höhe der Partikelemission und andererseits auf deren chemische Zusammensetzung. Die Erkenntnisse aus diesen Studien beziehen sich jedoch größtenteils auf den stationären Nennlast oder Teillastbetrieb. Der Praxisbetrieb hingegen ist nicht nur durch diese stationären Betriebszustände, sondern auch durch Anfahrvorgänge, Lastwechsel sowie Abschaltvorgänge charakterisiert. Daher wurde an der BIOENERGY 2020+ GmbH, Graz, Österreich, in Kooperation mit dem Institut für Prozess- und Partikeltechnik, Technische Universität Graz, ein Projekt zur Bestimmung und Charakterisierung partikelförmiger Emissionen über realitätsnahe Tageslastverläufe initiiert. Im Zuge des Projektes wurden für unterschiedliche Biomasse-Kleinfeuerungstechnologien Emissionsfaktoren bezüglich $\mathrm{CO}$, org.C und partikelförmige Emissionen über repräsentative Tageslastverläufe bestimmt. Des Weiteren wurden die Feinstaubemissionen bezüglich ihrer Korngrößenverteilung und speziell ihrer chemischen Zusammensetzung (anorganische Elemente, Kohlenstoff 


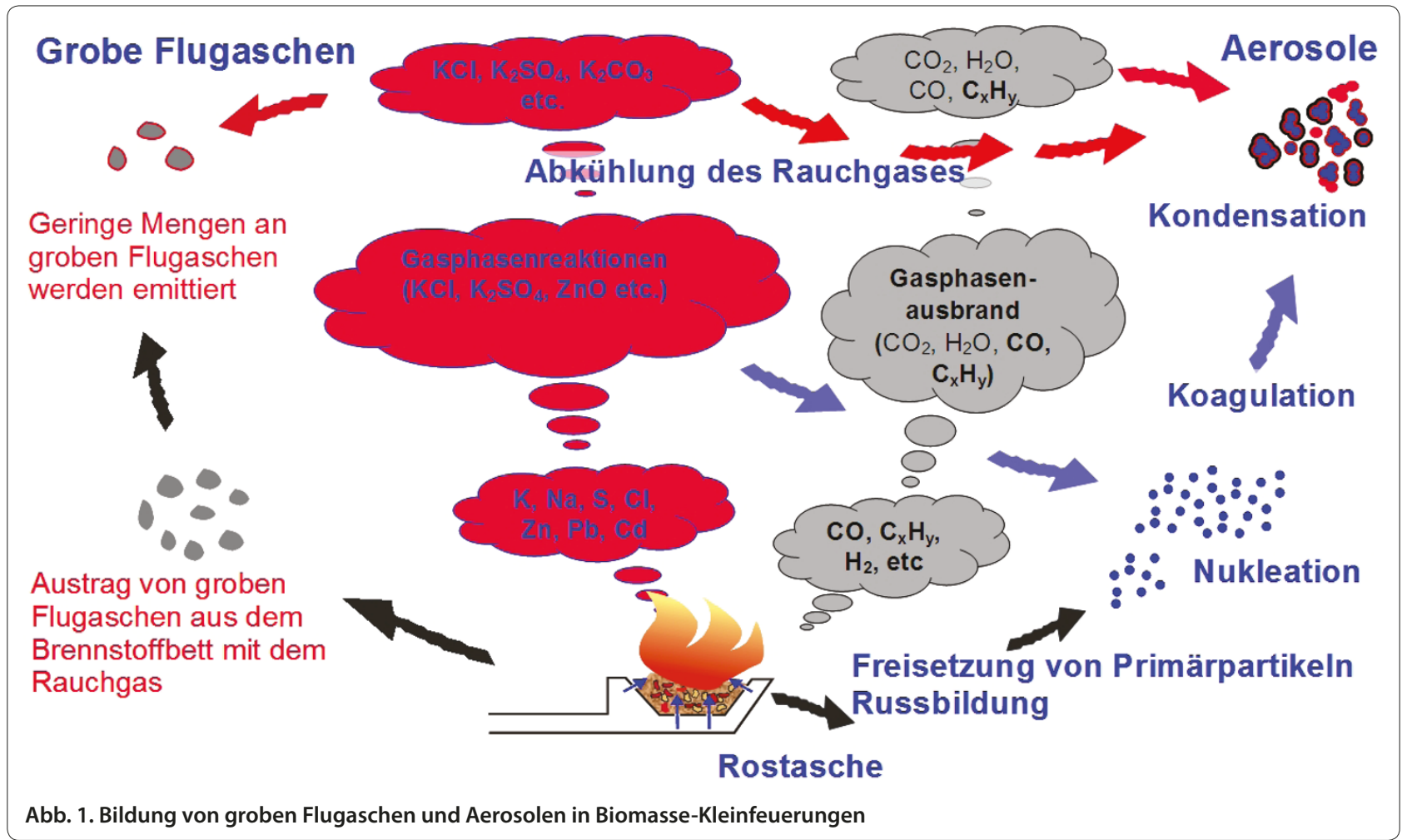

sowie polyzyklische aromatische Kohlenwasserstoffe) untersucht. Es wurden sowohl Altanlagen, die den Großteil des aktuellen Anlagenbestandes repräsentieren, als auch moderne Neuanlagen, die Stand der Technik sind, berücksichtigt. Aufbauend auf diesen Daten sollten die Zusammenhänge zwischen Anlagenbetrieb, partikelförmigen und gasförmigen Emissionen sowie der chemischen Zusammensetzung der partikelförmigen Emissionen möglichst geschlossen und nachvollziehbar dargestellt werden.

\section{Theoretische Hintergründe}

\subsection{Definition und Quellen für atmosphärische Stäube}

Da luftgetragene Partikel weder eine einheitliche Form noch eine einheitliche Dichte haben, wird für sie, um sie vergleichbar zu machen, der aerodynamische Durchmesser (ae.d.) angegeben. Dieser beschreibt ein kugelförmiges Teilchen mit einer Dichte von $1 \mathrm{~g} / \mathrm{cm}^{3}$, das gleich schnell wie das betrachtete Teilchen in Luft sinkt. Die in diesem Artikel erwähnten Partikelgrößen beziehen sich immer auf den aerodynamischen Durchmesser. Bei atmosphärischen Stäuben wird üblicherweise zwischen groben Partikeln oder Coarse Mode Particles (Partikel $>1 \mu \mathrm{m}$ bis ca. $100 \mu \mathrm{m}$ ) und feinen Partikeln oder Fine Mode Particles (Partikel $<1 \mu \mathrm{m}$ ) unterschieden [9]. Weitere verwendete Klassifizierungen umfassen den Begriff Gesamtstaub oder TSP (Total Suspended Particulate Matter = Gesamtheit aller Partikel in der Umgebungsluft) sowie die Bezeichnungen $\mathrm{PM}_{10}, \mathrm{PM}_{2,5}$ und $\mathrm{PM}_{1}(\mathrm{PM}=$ Particulate Matter oder Schwebstaub mit Durchmessern von $<10 \mu \mathrm{m},<2,5 \mu \mathrm{m}$ oder $<1 \mu \mathrm{m})$. Grobe Partikel entstehen bei mechanischen Prozessen, z.B. durch Abrieb und Aufwirbelungen aus dem Straßenverkehr, beim Umschlag von Schüttgütern, durch landwirtschaftliche Tätigkeiten und industrielle Prozesse sowie bei Vulkanausbrüchen, Wüstenstürmen, etc. Quellen für feine Partikel (Aerosole) hingegen sind thermische Prozesse in der Industrie, der Straßenverkehr (Dieselruß), die Landwirtschaft sowie der Hausbrand.

\subsection{Partikelbildung in Biomasse-Kleinfeuerungen}

Die bei der Verbrennung von festen Biomassebrennstoffen entstehenden Flugaschenemissionen lassen sich prinzipiell in zwei Kategorien, nämlich grobe Flugaschen (Partikel $>1 \mu \mathrm{m}$ ) und Aerosole (Partikel $<1 \mu \mathrm{m}$ ) unterteilen (siehe Abb. 1) [6,10].

Grobe Flugaschen entstehen durch Aufwirbelung von Brennstoff- oder Aschenpartikeln vom Brennstoffbett, die dann in weiterer Folge mit dem Rauchgas aus dem Feuerraum ausgetragen werden. Ein Teil dieser Flugaschen wird im Feuerraum und im Kessel wieder abgeschieden (so genannte Feuerraum- und Kesselaschen). Der im Rauchgas verbleibende Anteil verlässt die Feuerung als so genannte Flugaschenemission. Die Partikel weisen dabei üblicherweise Korngrößen von einigen $\mu \mathrm{m}$ bis zu $250 \mu \mathrm{m}$ auf und bestehen, ihrem Entstehungspfad entsprechend, aus typischen Grobaschenbildnern wie $\mathrm{Ca}, \mathrm{Si}, \mathrm{Mg}, \mathrm{Al}, \mathrm{Fe}$ und $\mathrm{K}$. 
Bei der deutlich komplexeren Aerosolbildung wird zwischen anorganischen Aerosolen sowie organischen Aerosolen und Russpartikeln differenziert. Anorganische Aerosole repräsentieren eine Fraktion, die in erster Linie von der chemischen Zusammensetzung des Brennstoffes beeinflusst wird. Bei der Verbrennung von Biomasse wird ein Teil der leichtflüchtigen Aschenbildner in die Gasphase freigesetzt, wobei, je nach Verbindung bzw. Element, die Freisetzungsraten stark variieren können. Für Biomasse-Kleinfeuerungen, die mit chemisch unbehandelter holzartiger Biomasse betrieben werden, sind hier vor allem $\mathrm{K}, \mathrm{S}, \mathrm{Cl}$ und $\mathrm{Zn}$ von Relevanz. In der Gasphase kommt es zu chemischen Reaktionen, die zur Bildung von Alkalimetallsulfaten, Alkalimetallchloriden und Alkalimetallkarbonaten sowie zur Bildung von Schwermetalloxiden führen. Überschreitet eine dieser Verbindungen ihren Sättigungsdampfdruck, so kommt es zu einem Phasenübergang in die flüssige bzw. feste Phase. Dieser Phasenübergang kann in Form von Nukleation (Bildung von neuen Partikeln mit Korngrößen von wenigen $\mathrm{nm}$ ) oder in Form von Kondensation auf Oberflächen (bestehende Partikel aber auch Wärmetauscheroberflächen) ablaufen. In weiterer Folge wachsen die so gebildeten Partikel durch Agglomerationseffekte an und erreichen am Kesselaustritt die für BiomasseKleinfeuerungen üblichen mittleren Korngrößen von ca. 0,1 bis 0,2 $\mu \mathrm{m}$. Durch den Zusammenhang zwischen der Bildung anorganischer Aerosole und dem eingesetzten Brennstoff liegt der Schluss nahe, dass bei annähernd vollständigem Gasphasenausbrand mit steigenden Konzentrationen an leicht flüchtigen Aerosolbildnern im Brennstoff die Feinstaubemissionen der Feuerung ansteigen.

Organische Aerosole und Rußemissionen resultieren aus der Kondensation von unverbrannten Kohlenwasserstoffen in Folge unzureichenden Gasphasenausbrands (organische Aerosole) sowie aus der unvollständigen Oxidation von Rußpartikeln. Bei optimiertem Gasphasenausbrand sowie bei entsprechend hohen Feuerraumtemperaturen lassen sich Emissionen an organischen Aerosolen und Rußemissionen annähernd vollständig vermeiden. Dies konnte im Zuge von Arbeiten zur Feinstaubbildung in Biomasse-Großfeuerungen eindeutig nachgewiesen werden [10]. In BiomasseKleinfeuerungen können jedoch häufig nicht während aller Betriebsphasen optimale Ausbrandbedingungen sichergestellt werden, weshalb den organischen Aerosolen und Rußemissionen ebenfalls Bedeutung zukommt.

\section{Angewandte Methodik}

\section{1 Überblick}

Es wurden Testläufe am Teststand der BIOENERGY 2020+ GmbH mit unterschiedlichen BiomasseKleinfeuerungen durchgeführt. Während der Testläufe wurden die wesentlichsten Betriebsparameter (Leistung,
Feuerraumtemperatur, Rauchgastemperatur am Kesselbzw. Ofenaustritt, Kaminzug usw.) sowie die bei der Verbrennung entstehenden gasförmigen (CO und org.C) und partikelförmigen Emissionen aufgezeichnet. Die Messungen der Betriebsparameter sowie der gasförmigen und partikelförmigen Emissionen erfolgte für die jeweiligen Feuerungen über typische Tageslastverläufe, die den Betrieb in der Praxis abbilden. Weiters wurden Feinstaubproben über den gesamten Tagesbetriebszyklus gezogen. Somit wurde sichergestellt, dass die gezogenen Partikelproben eine Mischprobe aus Emissionen des instationären wie auch des stationären Betriebs darstellen und daher mit den, durchschnittlich über einen Tagesbetriebszyklus im Feld emittierten, Partikeln vergleichbar sind. Mit den gezogenen Partikelproben wurden nachfolgend Analysen bezüglich ihrer chemischen Zusammensetzungen durchgeführt.

\subsection{Untersuchte Biomasse-Kleinfeuerungen}

Am Markt ist eine erhebliche Anzahl verschiedener Biomasse-Kleinfeuerungstechnologien von verschiedenen Anbietern verfügbar. Es wird generell zwischen handbeschickten und automatisch beschickten Feuerungen sowie zwischen Technologien mit erzwungener Durchströmung und Naturzug unterschieden. Es wird in der Regel holzartige Biomasse wie Stückholz, Hackgut oder Pellets als Brennstoff eingesetzt. Auch wenn in den letzten Jahren die Anzahl der jährlich neu installierten Pellet-, Hackgut- und Stückholzkessel gestiegen ist, dominieren alte Stückholzkessel mit unausgereifter Technologie neben Kaminöfen den aktuellen Anlagenbestand. Für die Messungen wurden die für Europa derzeit relevantesten Biomasse-Kleinfeuerungstechnologien ausgewählt:

- moderner Pelletkessel (entsprechend dem aktuellen Stand der Technik)

- moderner Hackgutkessel (entsprechend dem aktuellen Stand der Technik)

- moderner Stückholzkessel (entsprechend dem aktuellen Stand der Technik)

- alter Stückholzkessel (mit veralteter, den Anlagenbestand widerspiegelnder Technologie)

- moderner Kaminofen (entsprechend dem aktuellen Stand der Technik)

- Kaminofen (Billigprodukt mit veralteter Technologie)

- moderner Kachelofen (entsprechend dem aktuellen Stand der Technik)

Die Nennleistungen liegen für Anlagen mit erzwungener Durchströmung (Pellet-, Hackgut- und Stückholzkessel) zwischen 15 und $30 \mathrm{~kW}$, bei Kaminöfen im Bereich von $6 \mathrm{~kW}$ und beim Kachelofen bei rund $4 \mathrm{~kW}$. Sie repräsentieren somit die für die jeweilige Feuerungstechnologie in Europa üblichen Leistungsbereiche. Der Pellet- sowie der Hackgutkessel (20 sowie $30 \mathrm{~kW}$ ) verfügen über automatische Zündsysteme, 
gestufte Verbrennung, automatische Kesselreinigungssysteme sowie automatische Entaschungssysteme. Der Pelletkessel ist als Abwurffeuerung ausgeführt und besitzt eine wassergekühlte Brennkammer. Die automatische Verbrennungsregelung basiert auf einer Feuerraumtemperaturmessung. Der ebenfalls mit einer wassergekühlten Brennkammer ausgestatte Hackgutkessel ist als Unterschubfeuerung ausgeführt. Die Verbrennung wird über die Abgastemperatur sowie den Restsauerstoffgehalt (Lambda-Regelung) im Rauchgas automatisch geregelt.

Der moderne Stückholzkessel $(30 \mathrm{~kW})$ ist als Sturzbrandkessel mit unterem Abbrand ausgeführt. Die mikroprozessorgesteuerte automatische Verbrennungsregelung basiert auf einer Lambda-Regelung. Der alte Stückholzkessel mit einer Nennleistung von $15 \mathrm{~kW}$ ist durch einen seitlichen unteren Abbrand charakterisiert. Im Gegensatz zum modernen Stückholzkessel erfolgt die Verbrennungsregelung hier lediglich über ein thermomechanisches Luftklappensystem. Im Gegensatz zum automatisch beschickten Pellet- und Hackgutkessel werden beide Stückholzkessel manuell beschickt und entascht.

Der moderne Kaminofen $(6 \mathrm{~kW})$ repräsentiert den derzeitigen Stand der Technik und ist dem hohen Preissegment bei Kaminöfen (Qualitätsprodukt) zuzuordnen. Er verfügt über eine Brennraum- und Ausbrandzone. Die Regelung der Luftzuführung erfolgt manuell über einen Drehreglerknopf, der die Primärsowie die Scheibenluft regelt. Zusätzlich wurde ein Kaminofenbilligprodukt $(6,5 \mathrm{~kW}$, rund $€ 200)$ mit veralteter, unausgereifter Technologie eingesetzt. Sowohl die Primär- als auch die Sekundärluft werden hier über manuell zu betätigende Luftklappen eingestellt. Die Sekundärluft wird dabei über Düsen auf der Rückseite der Brennkammer zugeführt. Der getestete Kachelofen $(4,2 \mathrm{~kW})$ wurde nach den Richtlinien des Österreichischen Kachelofenverbandes ausgelegt. Die Verbrennungsluftzuführung erfolgt über einen Stehrost und die Fülltür.

\subsection{Tageslastverläufe}

Um praxisnahe Emissionsfaktoren für die CO-, org.Cund partikelförmigen Emissionen zu erhalten, wurden die zu untersuchenden Biomasse-Kleinfeuerungsanlagen unter Betriebsbedingungen getestet, die den Feld- bzw. Praxisbetrieb möglichst vollständig abbilden. Für die automatisch beschickten und automatisch geregelten Systeme (Pellet- und Hackgutkessel) wurden Tageslastverläufe abgefahren, die sowohl Anfahrvorgänge, stationäre Voll- und Teillastbetriebsphasen, Lastwechselvorgänge und Abfahrvorgänge beinhalteten. Bei den beiden Stückholzkesseln wurden die Füllräume zu Beginn mit Holz gefüllt, die Feuerung gezündet und bis zum
Abbrand des aufgegeben Brennstoffes betrieben. Nach Ende des Abbrandes wurde Brennstoff nachgelegt. Während bei den Testläufen mit dem modernen Stückholzkessel zwei Abbrände untersucht wurden, wurden aufgrund des geringeren Füllraumvolumens mit dem alten Stückholzkessel drei Abbrände jeweils bei Nennlast abgefahren. Typisch für den Betrieb von Naturzugöfen (Kamin- und Kachelöfen) ist der chargenweise Betrieb. Chargenweiser Betrieb (oder auch Batchbetrieb) bedeutet, dass der Brennraum manuell mit Brennstoff gefüllt und gezündet wird. Erst nach Abbrand der eingebrachten Brennstoffcharge wird der Brennraum wieder befüllt (das so genannte Nachlegen) und ein weiterer Abbrandzyklus beginnt. Ein Abbrandzyklus besteht immer aus mehreren Phasen, nämlich der Anbrennphase, der Hauptbrennphase und der Ausbrandphase. Bei den Testläufen mit den Kaminöfen wurden insgesamt 6 Abbrände in direkter Aufeinanderfolge bei Nennlast abgefahren. Der definierte Tageslastverlauf beim Kachelofen hingegen bestand technologiebedingt lediglich aus einem Abbrand bei Nennlast.

Generell erfolgten alle kontinuierlichen Messungen von Beginn des Testlaufs (Zünden) bis zum Ende des definierten Tageslastverlaufs (Erlöschen der Flamme). Die diskontinuierlichen Gesamtstaub- und Feinstaubmessungen erfolgten über den Tageslastverlauf verteilt so, dass der gesamte Testlauf abgedeckt wurde. Die Betriebs- und Emissionsdaten wurden in einem Intervall von 2 Sekunden aufgezeichnet, um nachfolgend entsprechende Auswertungen und Berechnungen durchführen zu können. Die so ermittelten Daten wurden für die jeweiligen Testläufe über den gesamten abgefahrenen Tageslastverlauf gemittelt, um repräsentative Emissionsfaktoren zu erhalten.

\subsection{Teststandaufbau und durchgeführte Messungen}

Das Schema des Teststandes sowie der eingesetzten Messanordnung sind in Abb. 2 dargestellt. Der Aufbau basiert auf Empfehlungen des ERA-NET Bioenergy Projekts BIOMASS PM [5] und folgt generell der EN 13240. Bei der Verbrennung von unbehandelter Biomasse in Naturzugöfen bzw. Feuerungen mit veralteter Technologie aber auch zeitweise beim Betrieb moderner Biomassekessel, treten Phasen mit erhöhten Emissionen kondensierbarer Kohlenstoffverbindungen auf. Diese gasförmigen Emissionen kondensieren im Realbetrieb in den Rauchgasleitungen zum Kamin, im Kamin oder am Kaminaustritt in der Atmosphäre und bilden ebenfalls Aerosole. Aus diesem Grund werden abweichend von der EN 13240 bei den Feinstaubmessungen Verdünnungseinheiten eingesetzt. Die Verdünnung erfolgt mit trockener und partikelfreier Druckluft. Das Verdünnungsverhältnis wird so eingestellt, dass das verdünnte Rauchgas eine Temperatur kleiner $40^{\circ} \mathrm{C}$ aufweist. Durch 


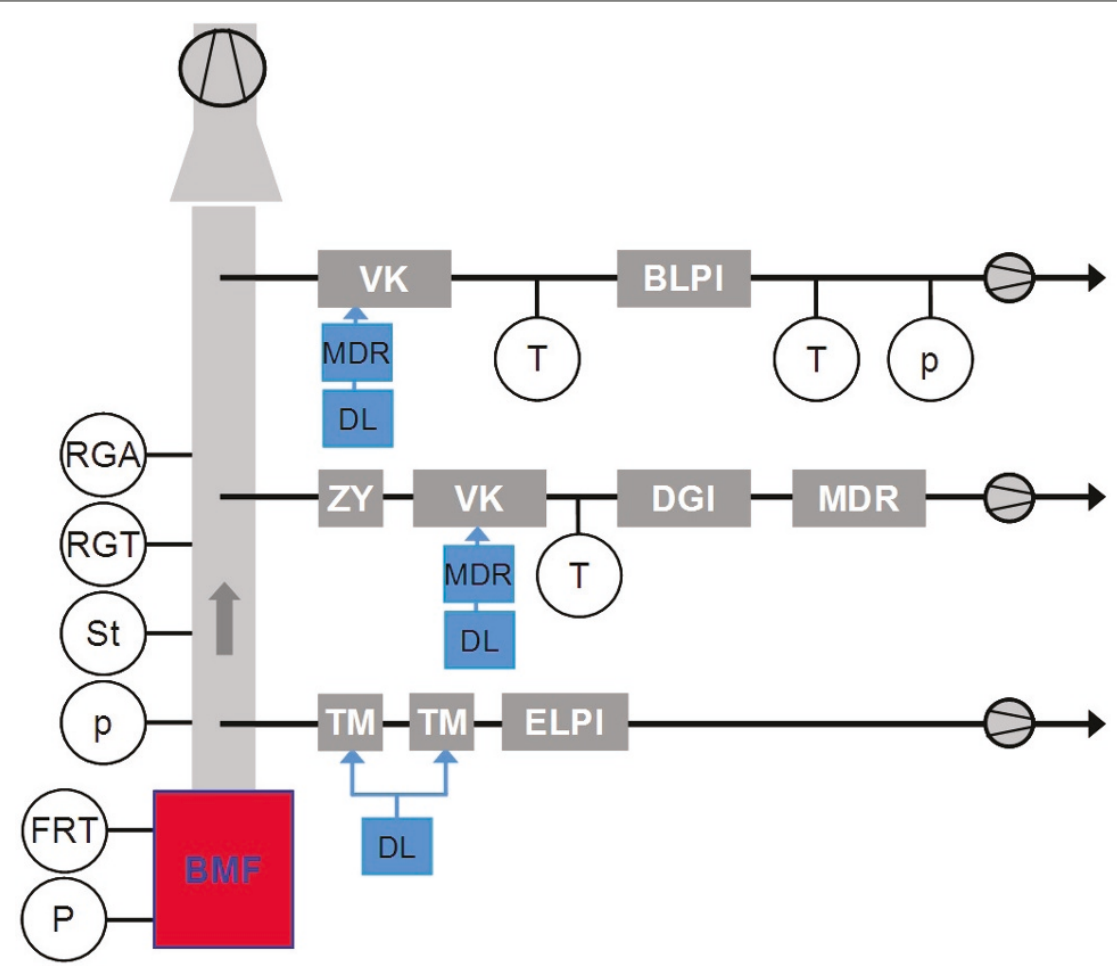

Abb. 2. Schematische Darstellung der Messanordnung. Erläuterung: Abkürzungen siehe Text

diese Maßnahmen soll sichergestellt werden, dass, unabhängig von der Rauchgastemperatur an der Messstelle und der Ausbrandqualität, Partikelkonzentrationen, die denen am Kaminaustritt entsprechen, gemessen werden.

Im Folgenden wird die in Abb. 2 dargestellte Messanordnung kurz erläutert. BMF stellt die zu untersuchende Biomasse-Kleinfeuerung dar. Bezüglich des Feuerungsbetriebes werden die Feuerraumtemperatur (FRT) sowie bei Kesseln die Anlagenleistung (P) kontinuierlich erfasst und aufgezeichnet. Im unverdünnten Rauchgas nach Kessel- bzw. Ofenaustritt werden der Kaminzug (p), die Rauchgastemperatur (RGT), sowie die Konzentrationen an $\mathrm{O}_{2}, \mathrm{CO}, \mathrm{CO}_{2}$ und org. $\mathrm{C}$ mittels Rauchgasanalysatoren (RGA) kontinuierlich aufgezeichnet. Die Bestimmung der Gesamtstaubgehalte (St) erfolgt diskontinuierlich mit dem Verfahren nach VDI 2066.

Zur Bestimmung der Konzentration und Korngrößenverteilung der Feinstaubemissionen im verdünnten Rauchgas werden zwei diskontinuierliche Messverfahren (Bestimmung mittels Dekati Gravimetric Impactor sowie Berner-Typ Niederdruck-Kaskadenimpaktor) sowie ein kontinuierliches Messverfahren (Bestimmung mittels elektrischem Niederdruck-Kaskadenimpaktor) eingesetzt. Für die kontinuierliche Bestimmung der Konzentration und Korngrößenverteilung der Feinstaubemissionen mit einem elektrischen Niederdruck-Kaskadenimpaktor (ELPI) wird das Rauchgas dem Rauchgaskanal entnommen und mit Hilfe zweier in Serie geschalteter turbulenten
Mischer (TM) verdünnt. Die Verdünnungscharakteristik der turbulenten Mischer, die vom Druck der Verdünnungsluft abhängt, wurde im Zuge von Vorversuchen ermittelt. Das Verdünnungsverhältnis lässt sich aus dem Volumenstrom durch den ELPI, der mittels kritischer Düse geregelt wird, und der Verdünnungscharakteristik der turbulenten Mischer berechnen. Die von den Biomasse-Kleinfeuerungen über den Tageslastverlauf emittierten Feinstaubemissionen werden aus den Daten der kontinuierlichen ELPI-Messung berechnet. Da sich die Daten welche vom ELPI generiert werden auf die Partikelanzahl beziehen, wird ein Korrekturfaktor für die Dichte und Partikelform der Feinstaubpartikel berücksichtigt, um massenbezogene Daten zu erhalten. Aufgrund der Heterogenität der Partikel ist es nicht möglich durch theoretische Überlegungen verlässliche Korrekturfaktoren $\mathrm{zu}$ bestimmen. Deshalb werden parallel zu den ELPI-Messungen, Messungen mit einem Berner-Typ Niederdruck-Kaskadenimpaktor (BLPI) und Dekati Gravimetric Impactor (DGI) durchgeführt, um aus diesen massenbezogenen Ergebnissen einen mittleren Korrekturfaktor zu berechnen sowie die Konzentration und Korngrößenverteilung der Feinstaubemissionen zu bestimmen. Hierfür wird ein Teilstrom über eine Entnahmesonde und einen beheizten $\mathrm{PM}_{10}$-Zyklon (ZY) mit einer Pumpe dem Rauchgasstrom entnommen und im Verdünnungskühler (VK) mit gefilterter und getrockneter Druckluft (DL) verdünnt. Die Messung der 
Verdünnungsluftmenge erfolgt über einen Massedurchflussregler (MDR). Am Austritt aus dem Verdünnungskühler wird die Temperatur ( $\mathrm{T}$ ) des verdünnten Rauchgases gemessen. Das verdünnte Rauchgas wird durch den Dekati Gravimetric Impactor (DGI) gesaugt, wobei der Volumenstrom über einen weiteren Massedurchflussregler (MDR) geregelt wird. Das Verdünnungsverhältnis wird aus den Messdaten der beiden Massedurchflussregler berechnet. Eine weitere Teilgasstromentnahme mit nachfolgender Verdünnung über einen Verdünnungskühler (VK) erfolgt für die Bestimmung der Konzentration und Korngrößenverteilung der Feinstaubemissionen mit einem BLPI. Hier wird der Verdünnungsluftstrom (DL) ebenfalls mit einem Massedurchflussregler (MDR) geregelt und die Temperatur des verdünnten Rauchgases (T) kontinuierlich erfasst. Das Verdünnungsverhältnis wird über den Rauchgasvolumenstrom über den BLPI, der über eine kritische Düse geregelt wird, und den Verdünnungsluftstrom berechnet. Zusätzlich wird auch der Unterdruck (p) und die Rauchgastemperatur (T) nach dem BLPI gemessen. Alle Betriebsdaten der Verdünnungsstrecken (Verdünnungsluftmassenströme, Temperaturen des verdünnten Rauchgases) werden kontinuierlich aufgezeichnet. Bei jedem Testlauf wird der eingesetzte Brennstoff und der entstandene Verbrennungsrückstand (Rost- und Bettasche) beprobt sowie bei manuell beschickten Feuerungen zusätzlich gewogen.

Der eingesetzte Brennstoff wird nasschemisch hinsichtlich des Wassergehalts (ÖNORM CEN/TS 14774), Aschegehalts (ÖNORM CEN/TS 14775), der Konzentrationen an C, H, N (ÖNORM CEN/TS 15104), Si, Ca, $\mathrm{Mg}, \mathrm{Mn}, \mathrm{K}, \mathrm{Na}, \mathrm{Zn}, \mathrm{S}$ (mehrstufiger Druckaufschluss mit $\mathrm{HNO}_{3} / \mathrm{HF} / \mathrm{H}_{3} \mathrm{BO}_{3}$ und Elementbestimmung mittels ICPOES) sowie Cl (ÖNORM CEN/TS 15289) analysiert. Bei ausgewählten Feinstaubproben werden die Konzentrationen von Haupt- und Nebenelementen mittels mehrstufigen Druckaufschluss und Detektion mittels ICP-OES oder ICP-MS sowie die Gehalte an organischem Kohlenstoff (OC) und elementarem Kohlenstoff (EC) mit einem "Carbon/Hydrogen/Moisture Determinator" der Firma LECO, Modell RC-612, bestimmt. Der Einsatz des DGI in Kombination mit einem Verdünnungskühler eröffnet zusätzlich zur Bestimmung der Konzentration und Korngrößenverteilung der Feinstaubemissionen die Möglichkeit während einer DGI-Messung rund 20 mg Feinstaub zu sammeln und somit, je nach Tageslastverlauf und Höhe der partikelförmigen Emissionen, bis zu 100 mg Feinstaub zu generieren. Mit dieser Partikelmasse war es anschließend möglich einerseits die Gehalte an polyzyklischen aromatischen Kohlenwasserstoffen (PAK) zu bestimmen und anderseits toxikologische Untersuchungen (toxikologische Untersuchungen sind noch nicht abgeschlossen und können deshalb in diesem Artikel nicht präsentiert werden) mit den Feinstaubproben durchzuführen, um das vom Feinstaub ausgehende Gesundheitsgefährdungspotential zu bewerten.

\subsection{Eingesetzter Brennstoff}

Für die Testläufe wurden Weichholzpellets gemäß ÖNORM M 7135, Hackgut gemäß ÖNORM M 7133 sowie Stückholz (Buche) gemäß ÖNORM M 7132 und ÖNORM CEN/TS 14961 eingesetzt. Es wurden jeweils mehrere Teilproben des Brennstoffes gezogen und zu einer Testlauf-Mischprobe vereint, die dann analysiert wurde. Alle eingesetzten Brennstoffe entsprachen den Anforderungen der einschlägigen Normen. Die Ergebnisse der nasschemischen Analysen sind in Tabelle 1 zusammengefasst. Der Wassergehalt der Pellets (Pe-m) betrug 7,4 Gew.\% FS. Die Wassergehalte des eingesetzten Hackguts (HG-m) (<13 Gew.\% FS) und des Stückholzes (alle mit SH gekennzeichneten Sortimente) $(<12$ Gew.\% FS) sind als vergleichsweise gering $\mathrm{zu}$ bezeichnen. Die Aschegehalte steigen von Pellets und Hackgut $(0,4$ bzw. 0,3 Gew.\% TS) zum Stückholz (0,6 bis 1,9 Gew.\% TS), wobei $\mathrm{Ca}, \mathrm{Si}, \mathrm{Mg}$ und $\mathrm{K}$ die relevantesten Aschebildner sind. Die Konzentrationen von $\mathrm{C}, \mathrm{H}$ und $\mathrm{N}$ liegen in einem für chemisch unbehandelte holzartige Biomasse typischen Bereich und weichen nur geringfügig voneinander ab. Die S- und Cl-Konzentrationen liegen für die eingesetzten Brennstoffe ebenso im für Holzbrennstoffe repräsentativen Bereich. $\mathrm{K}$ ist bei allen eingesetzten Brennstoffen das am stärksten konzentrierte aerosolbildende Element. Die K-Konzentrationen liegen für Pellets und Hackgut auf einem vergleichbaren Niveau (418 bzw. $401 \mathrm{mg} / \mathrm{kg}$ TS) und sind für das eingesetzte Stückholz um den Faktor 3 bis 4 höher $(1.375$ bis $1.790 \mathrm{mg} / \mathrm{kg}$ TS), was auf den höheren Rindenanteil und den höheren Aschegehalt von Hartholz im Vergleich zu Weichholz zurückzuführen ist. Die anderen aerosolbildenden Elemente $(\mathrm{Na}, \mathrm{Zn}$ und $\mathrm{Pb})$ weisen für alle untersuchten Brennstoffe verglichen mit $\mathrm{K}$ wesentlich geringere Konzentrationen auf und liegen auf einem ähnlichen niedrigen Niveau. Die Summe der Aerosolbildner (K, $\mathrm{Na}, \mathrm{Pb}$ und $\mathrm{Zn}$ ) sind für Pellets und Hackgut ähnlichen (444 bzw. $410 \mathrm{mg} / \mathrm{kg}$ TS) während sie für das Stückholz deutlich höher liegen (1.380 bis $1.794 \mathrm{mg} / \mathrm{kg}$ TS).

\section{Ergebnisse und Diskussion}

4.1 Ermittelte Betriebsdatenverläufe sowie gas- und partikelförmige Emissionen

Bei instationärem Betrieb (Hochfahren, Lastwechselbetrieb, Abfahren) treten bei allen automatisch geregelten Feuerungen (Pellets-, Hackgut- und Stückholzkessel) kurzfristige gas- und partikelförmige Emissionsspitzen auf, welche im Hochfahrbetrieb am deutlichsten ausgebildet sind. Beim modernen Stückholzkessel sind diese Emissionsspitzen stärker als beim Pellet- und Hackgutkessel. Bei Erreichen stationärer Betriebsbedingungen 
Tabelle 1. Chemische Zusammensetzung der eingesetzten Brennstoffe für die Testläufe

\begin{tabular}{|c|c|c|c|c|c|c|c|c|}
\hline Parameter & Einheit & Pe-m & HG-m & SH-m & $\mathrm{SH}-\mathrm{a}$ & Ka-m & Ka-a & Ko-m \\
\hline Brennstoff & & Pellets & Hackgut & Stückholz & Stückholz & Stückholz & Stückholz & Stückholz \\
\hline Holzart & & Weichholz & Weichholz & Hartholz & Hartholz & Hartholz & Hartholz & Hartholz \\
\hline Wassergehalt & Gew. \% FS & 7,4 & 12,7 & 8,7 & 12,0 & 8,5 & 9,4 & 9,1 \\
\hline Aschegehalt & Gew. \% TS & 0,4 & 0,3 & 0,6 & 1,0 & 0,7 & 0,6 & 1,9 \\
\hline C & Gew. \% TS & 49,9 & 49,0 & 48,3 & 48,6 & 48,3 & 48,3 & 48,4 \\
\hline $\mathrm{H}$ & Gew. \% TS & 6,1 & 6,3 & 6,1 & 6,2 & 6,3 & 6,1 & 6,0 \\
\hline N & Gew. \% TS & 0,1 & 0,1 & 0,1 & 0,2 & 0,2 & 0,1 & 0,2 \\
\hline S & $\mathrm{mg} / \mathrm{kg}$ TS & 61,1 & 56,5 & 103,0 & 131,0 & 89,3 & 89,5 & 137,5 \\
\hline $\mathrm{Cl}$ & $\mathrm{mg} / \mathrm{kg}$ TS & 12,0 & 12,0 & 11,1 & 18,0 & 14,2 & 10,6 & 12,8 \\
\hline $\mathrm{Ca}$ & $\mathrm{mg} / \mathrm{kg}$ TS & 888,0 & 861,0 & $1.140,0$ & $2.530,0$ & $1.090,0$ & $1.100,0$ & $5.955,0$ \\
\hline $\mathrm{Si}$ & $\mathrm{mg} / \mathrm{kg} \mathrm{TS}$ & 270,0 & $<100,0$ & $<200,0$ & $<200,0$ & $<200,0$ & $<200,0$ & 275,0 \\
\hline$M g$ & $\mathrm{mg} / \mathrm{kg}$ TS & 107,0 & 182,0 & 506,5 & 527,0 & 221,0 & 518,0 & 280,5 \\
\hline K & $\mathrm{mg} / \mathrm{kg}$ TS & 418,0 & 401,0 & $1.375,0$ & $1.440,0$ & $1.790,0$ & $1.460,0$ & $1.580,0$ \\
\hline $\mathrm{Na}$ & $\mathrm{mg} / \mathrm{kg}$ TS & 14,8 & 2,7 & 1,9 & 2,0 & 2,0 & 2,0 & 3,7 \\
\hline $\mathrm{Pb}$ & $\mathrm{mg} / \mathrm{kg}$ TS & $<5,0$ & 0,1 & $<5,0$ & $<5,0$ & $<5,0$ & $<5,0$ & $<5,0$ \\
\hline Zn & $\mathrm{mg} / \mathrm{kg}$ TS & 11,4 & 6,2 & 3,5 & 3,0 & 2,1 & 2,3 & 2,6 \\
\hline $\begin{array}{l}\text { Summe } \\
\text { Aerosolbildner } \\
\text { (K+Na+Pb+Zn) }\end{array}$ & $\mathrm{mg} / \mathrm{kg}$ TS & 444,2 & 410,0 & $1.380,4$ & $1.445,0$ & $1.794,1$ & $1.465,3$ & $1.586,3$ \\
\hline
\end{tabular}

sinken die Emissionen aufgrund nahezu vollständigen Gasphasenausbrandes deutlich ab. Lastwechselvorgänge auf Teillast bewirken neben dem Leistungsabfall eine Zunahme des $\mathrm{O}_{2}$-Gehaltes im Rauchgas und eine Abnahme der Feuerraumtemperatur. Die gasförmigen und partikelförmigen Emissionen verändern sich in diesen Phasen nur unwesentlich. Im Teillastbetrieb sind die $\mathrm{O}_{2}$-Gehalte im Vergleich zum Nennlastbetrieb erhöht. Die Zunahme des Luftüberschusses bewirkt niedrigere Temperaturen im Feuerraum und somit verschlechterte Randbedingungen für den Gasphasenausbrand. Deshalb sind die gasförmigen Emissionen höher als im Nennlastbetrieb, liegen aber immer noch auf einem niedrigen Niveau. Die $\mathrm{PM}_{1}$-Emissionen steigen im Vergleich zu den Nennlastphasen leicht an und lassen auf die Bildung von organischen Aerosolen und Rußemissionen schließen. Während der Abfahrvorgänge kommt es, verbunden mit dem Ansteigen der $\mathrm{O}_{2}$ Konzentrationen im Abgas sowie dem Absinken der Feuerraumtemperaturen, wiederum zu einem Ansteigen der gasförmigen und partikelförmigen Emissionen. In Abb. 3 ist der Verlauf der Betriebsdaten sowie der gasund partikelförmigen Emissionen für einen Testlauf mit dem modernen Pelletkessel (Nennleistung: $20 \mathrm{~kW}$ ) dargestellt.

Der Betrieb von Naturzugöfen (Kaminöfen und Kachelöfen) ist durch eine Anbrenn-, Hauptbrenn- und Ausbrandphase charakterisiert. Die Anbrennphase ist durch erhöhte gasförmige und partikelförmige Emissionen gekennzeichnet, die in erster Linie auf die vergleichsweise niedrigen Feuerraumtemperaturen in diesen Zeitabschnitten zurückzuführen sind. Mit steigenden Feuerraumtemperaturen verbessert sich die Ausbrandqualität und die gas- und partikelförmigen Emissionen sinken deutlich. Es folgt eine Phase mit durchwegs niedrigeren Emissionen, die so genannte Hauptbrennphase. Nach Freisetzung aller flüchtigen brennbaren Komponenten aus dem Brennstoff kommt es letztendlich während der so genannten Ausbrandphase zur Verbrennung der verbleibenden Holzkohle. Diese Phase ist durch sinkende Feuerraumtemperaturen und steigende $\mathrm{O}_{2}$-Konzentrationen gekennzeichnet, was auch zu erhöhten COEmissionen führt. Ein für den modernen Kaminofen typischer Verlauf der Betriebsdaten sowie der gas- und partikelförmigen Emissionen ist in Abb. 4 dargestellt. Es wurden insgesamt 6 Batches (ein so genannter Anheizbatch und fünf Batches), deren Beginn bzw. Ende durch die senkrechten schwarz-strichlierten Linien gekennzeichnet ist, bei Nennleistung $(6 \mathrm{~kW})$ abgefahren. Die während eines Batches auftretenden Phasen (Anbrenn-, Hauptbrenn- und Ausbrandphase) sind exemplarisch während Batch 3 durch die senkrechten rot-strichlierten Linien dargestellt.

\subsection{Durchschnittliche Emissionsfaktoren über den Tageslastverlauf}

Zur Bewertung der Emissionsfaktoren bezüglich der $\mathrm{CO}$, org.C- und Feinstaubemissionen wurden mit Ausnahme 

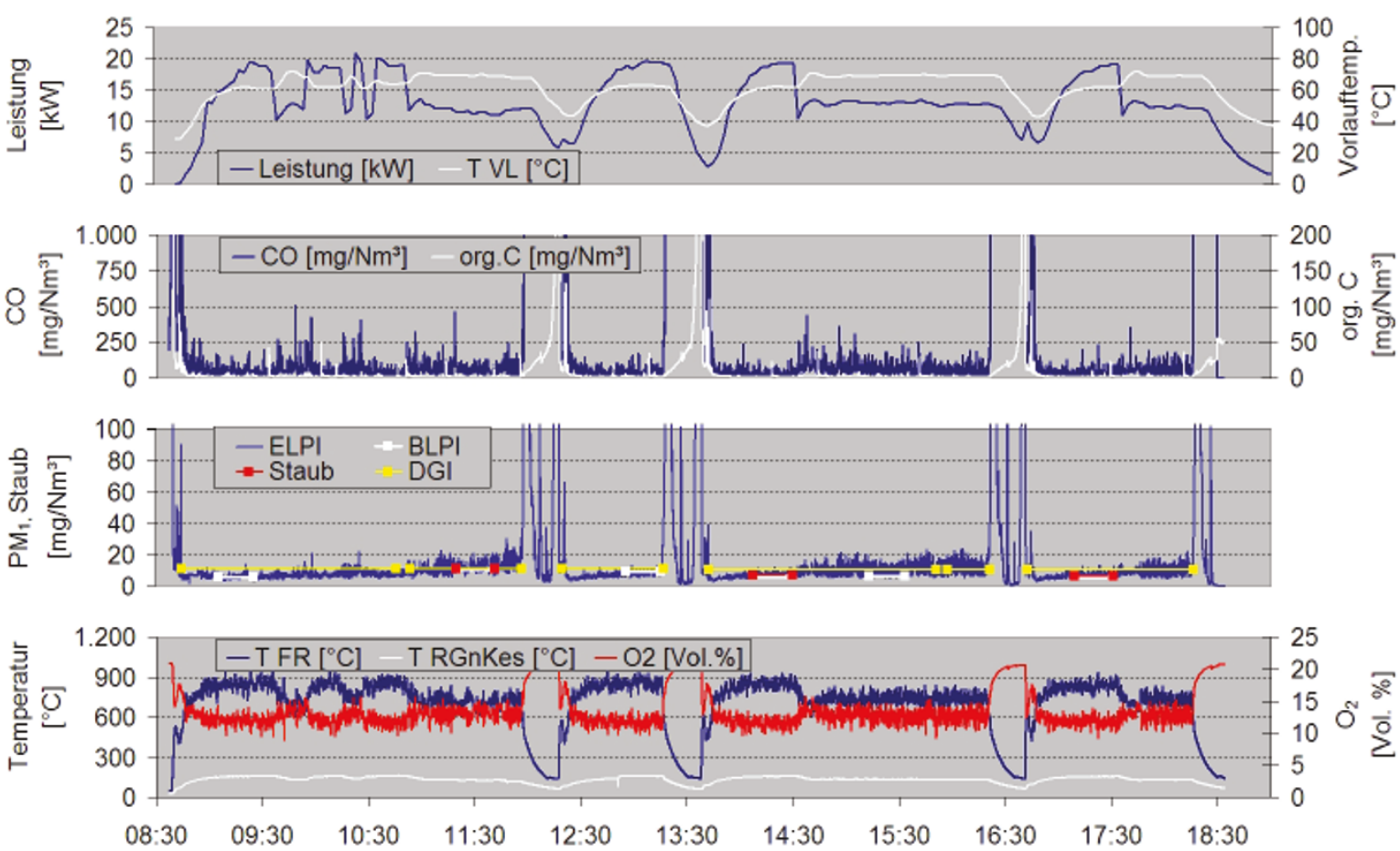

Abb. 3. Betriebsdatenverläufe sowie gas- und partikelförmige Emissionen während eines Testlaufs mit dem Pelletkessel (Nennleistung: $20 \mathrm{~kW})$
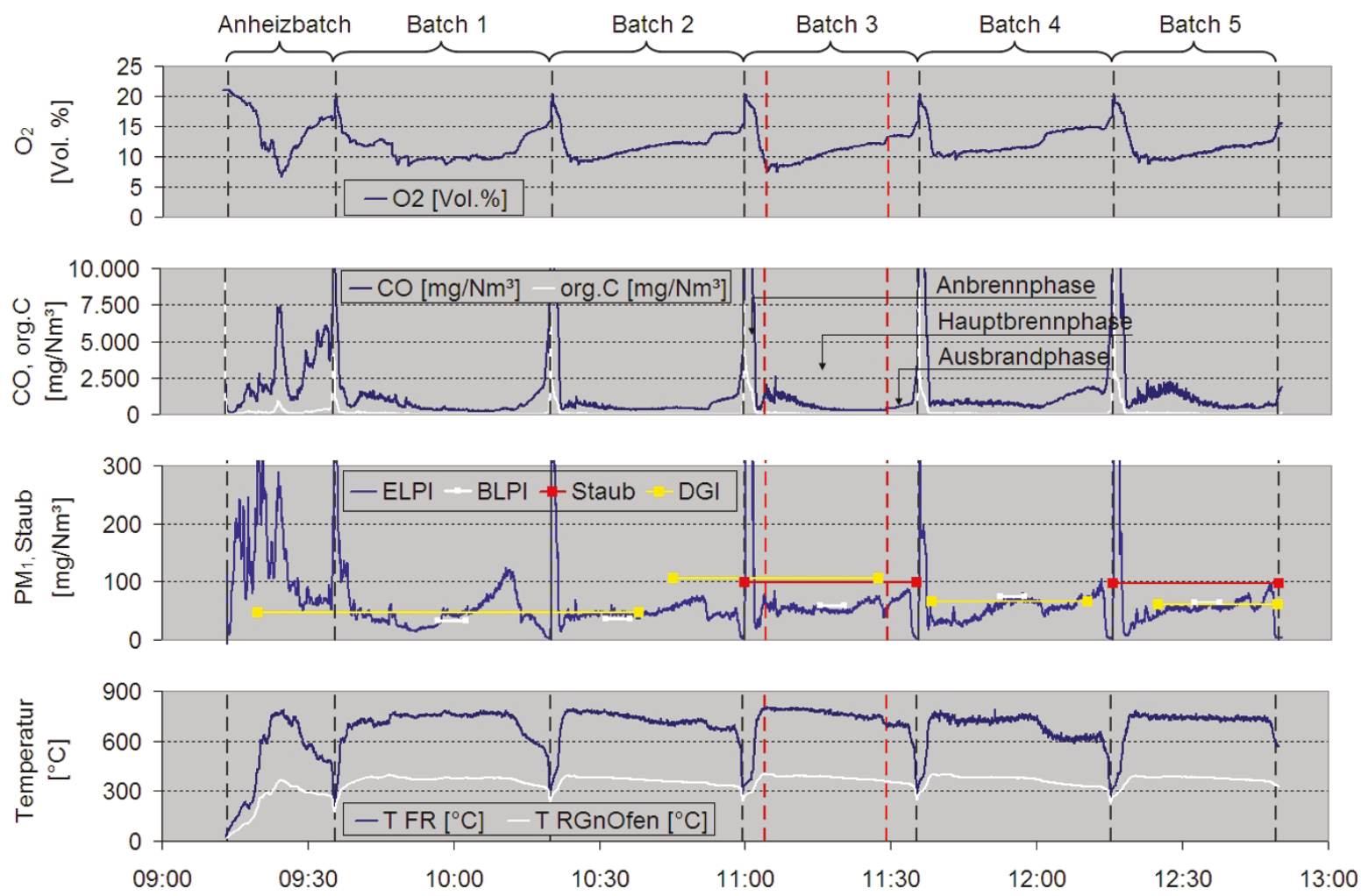

Abb. 4. Betriebsdatenverläufe sowie gas- und partikelförmige Emissionen während eines Testlaufs mit dem modernen Kaminofen (Nennleistung: $6 \mathrm{~kW}$ ) 


\begin{tabular}{|c|c|c|c|c|c|}
\hline Feuerung & Testlauf & $\begin{array}{c}\mathrm{O}_{2} \\
\text { [Vol.\%] }\end{array}$ & $\begin{array}{c}\mathrm{CO} \\
{[\mathrm{mg} / \mathrm{MJ}]}\end{array}$ & $\begin{array}{c}\text { org.C } \\
{[\mathrm{mg} / \mathrm{MJ}]}\end{array}$ & $\begin{array}{c}\mathrm{PM}_{1} \\
{[\mathrm{mg} / \mathrm{MJ}]}\end{array}$ \\
\hline \multirow[t]{2}{*}{ Pe-m } & 1 & 12,6 & 47,1 & 2,5 & 6,2 \\
\hline & 2 & 12,5 & 45,4 & 1,7 & 6,0 \\
\hline \multirow[t]{2}{*}{$\mathrm{Hg}-\mathrm{m}$} & 1 & 12,2 & 168,1 & 3,0 & 15,3 \\
\hline & 2 & 12,1 & 182,2 & 5,4 & 13,6 \\
\hline \multirow[t]{2}{*}{ Sh-m } & 1 & 8,6 & 700,4 & 78,7 & 14,2 \\
\hline & 2 & 8,6 & 793,1 & 62,4 & 17,6 \\
\hline \multirow[t]{6}{*}{ Ko-m } & 1 & 15,6 & $1.180,0$ & 68,2 & 45,4 \\
\hline & 2 & 15,4 & $1.282,0$ & 52,6 & 30,0 \\
\hline & 3 & 15,1 & $1.032,0$ & 48,7 & 37,8 \\
\hline & 4 & 15,3 & $1.159,9$ & 36,4 & 18,4 \\
\hline & 5 & 15,5 & 875,6 & 36,5 & 22,1 \\
\hline & 6 & 15,4 & $1.114,8$ & 53,3 & 24,0 \\
\hline \multirow[t]{2}{*}{$\mathrm{Ka}-\mathrm{m}$} & 1 & 12,5 & $1.048,2$ & 94,2 & 47,2 \\
\hline & 2 & 12,1 & $1.035,6$ & 95,5 & 46,1 \\
\hline \multirow[t]{2}{*}{ Ка-а } & 1 & 10,8 & $2.355,4$ & 223,9 & 74,2 \\
\hline & 2 & 11,1 & $2.084,6$ & 185,7 & 55,5 \\
\hline \multirow[t]{2}{*}{ Sh-a } & 1 & 11,3 & $12.632,3$ & $1.143,8$ & 106,1 \\
\hline & 2 & 11,0 & $8.969,4$ & 650,8 & 98,6 \\
\hline
\end{tabular}

Erläuterung: Mittelwerte über den jeweiligen Tageslastverlauf; $\mathrm{O}_{2}$ -

Konzentrationen bezogen auf trockenes Rauchgas

des Kachelofens jeweils zwei Testläufe pro Feuerungstyp über den definierten Tageslastverlauf durchgeführt und bewertet. Mit dem Kachelofen wurden insgesamt 6 Testläufe durchgeführt, um eine ausreichende Anzahl repräsentativer Abbrände zu erhalten. In Tabelle 2 sind die über die Tageslastverläufe gemittelten gas- und partikelförmigen Emissionen zusammengefasst.

Es ist eindeutig zu erkennen, dass sowohl die gasförmigen als auch die partikelförmigen Emissionen über den gesamten Tageslastverlauf innerhalb der einzelnen Feuerungen gut miteinander vergleichbar sind. Weiters wurde anhand der durchgeführten Testläufe belegt, dass es zwischen den einzelnen Feuerungstechnologien starke Unterschiede hinsichtlich der Höhe der Emissionen gibt. Die untersuchten Biomasse-Kleinfeuerungen können in drei Gruppen eingeteilt werden. Die erste Gruppe beinhaltet moderne automatische Kesselanlagen (moderner Pellet-, Hackgut- und Stückholzkessel). Diese Anlagen repräsentieren den aktuellen Stand der Technik und sind durch die geringsten gas- und partikelförmigen Emissionen der untersuchten Feuerungstechnologien charakterisiert. Die zweite Gruppe beinhaltet moderne Naturzugöfen (moderner Kaminofen und Kachelofen). Beide Systeme zeigen vergleichbare CO-Emissionen,

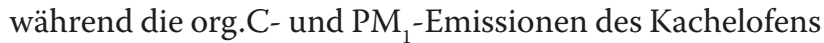
etwas geringer sind als die des Kaminofens. In die dritte
Gruppe fallen die Feuerungen mit veralteter Technologie (alter Stückholzkessel und Kaminofenbilligprodukt mit veralteter Technologie). Die Feinstaubemissionen des Kaminofenbilligprodukts liegen 20 bis $60 \%$ über jenen des modernen Kaminofens. Die mit Abstand höchsten gasförmigen und partikelförmigen Emissionen wurden bei den Testläufen mit dem alten Stückholzkessel festgestellt. Sie sind mit Literaturdaten [11, 12] gut vergleichbar und spiegeln das Betriebsverhalten derartiger Altanlagen gut wider.

In Abb. 5 und Abb. 6 sind Korrelationen zwischen verschiedenen Emissionsparametern (gemittelte Emissionsdaten über die jeweiligen Testläufe) dargestellt. Schlechter Gasphasenausbrand resultiert aus zu geringen Feuerraumtemperaturen sowie aus schlechter Durchmischung der Rauchgase mit der Verbrennungsluft und führt zu erhöhten gas- und partikelförmigen Emissionen. Die Auswirkungen schlechter werdenden Gasphasenausbrands lassen sich anhand des Vergleichs der $\mathrm{CO}$ - und org.C-Emissionen bzw. $\mathrm{PM}_{1}$-Emissionen gut darstellen. Mit steigenden CO-Emissionen steigen auch die org.CEmissionen (Abb. 5, $\mathrm{R}^{2}=0,9427$ ) bzw. $\mathrm{PM}_{1}$-Emissionen (Abb. 6, $\left.R^{2}=0,8503\right)$ statistisch signifikant $(p<0,05)$ an. Weiters wurde beim Vergleich der PM $_{1}$-Emissionen mit den org.C-Emissionen (Ergebnisse nicht dargestellt), wie $\mathrm{zu}$ erwarten war, eine hohe Korrelation $\left(R^{2}=0,8215\right)$ festgestellt, die statistisch als Trend vorliegt $(\mathrm{p}<0,1)$.

\subsection{Chemische Charakterisierung der Feinstaubemissionen} Ausgewählte, mit den BLPI gesammelte $\mathrm{PM}_{1}$-Proben, wurden nasschemisch analysiert. Es wurde anhand von Hochtemperaturgleichgewichtsberechnungen sowie XRDAnalysen (Röntgendiffraktometrie) nachgewiesen, dass $\mathrm{S}$ und $\mathrm{Cl}$ mit Alkalimetallen ( $\mathrm{K}$ und $\mathrm{Na}$ ) reagieren und Alkalimetallsulfate bzw. Alkalimetallchloride bilden wobei $\mathrm{K}$ aufgrund der höheren Freisetzungsrate der relevanteste Feinstaubbildner ist [10,13-17]. Der verbleibende Rest der Alkalimetalle (hauptsächlich K) wird als Alkalimetallkarbonat gebunden. Basierend auf den Ergebnissen der chemischen Elementanalysen wurde die Zusammensetzung der Feinstaubemissionen hinsichtlich ihrer Hauptverbindungen kalkuliert. Hierbei wurde zuerst die Konzentration der Alkalimetallsulfate unter der Annahme, dass der gesamte Schwefel als Sulfat gebunden wird, berechnet. Danach wurde unter derselben Annahme für Chlor (das gesamte Chlor wird als Chlorid gebunden) die Alkalimetallchloridkonzentration berechnet. Die nicht als Sulfat oder Chlorid gebundenen Alkalimetalle wurden als Alkalimetallkarbonate berücksichtigt. Da die Schwermetallkonzentrationen in chemisch unbehandelter Biomasse als gering einzustufen sind, weisen auch die Feinstaubkonzentrationen nur geringe Schwermetallanteile (hauptsächlich $\mathrm{Zn})$ auf. Die Ca-, Si- und $\mathrm{Mg}$-Anteile in den 


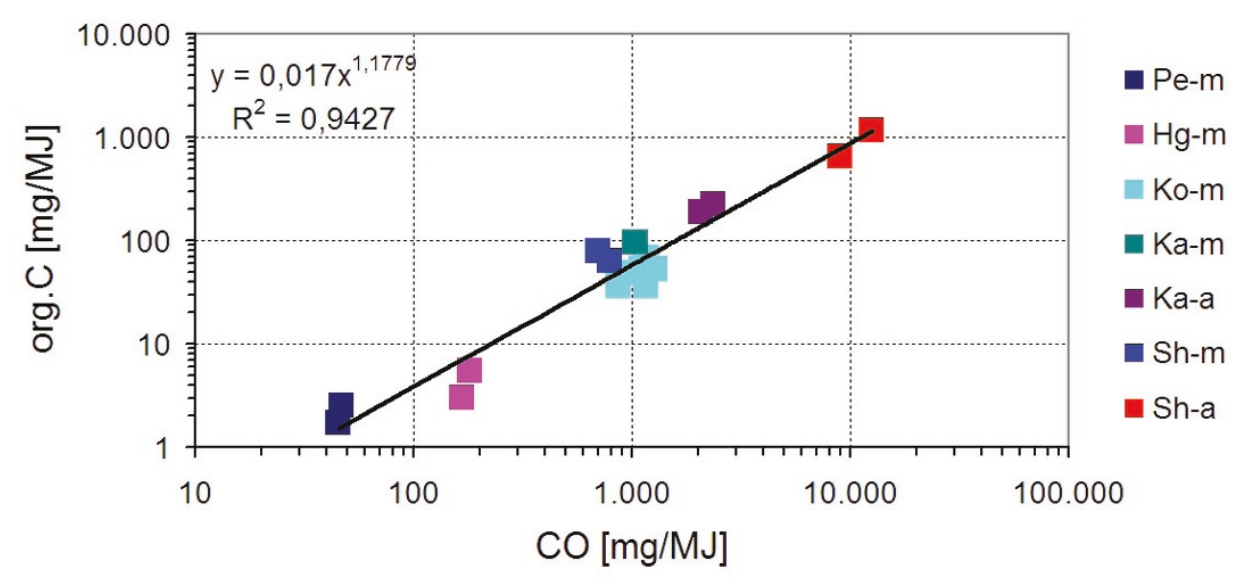

Abb. 5. Korrelation der CO- und der org.C-Emissionen für alle untersuchten Biomasse-Kleinfeuerungsanlagen

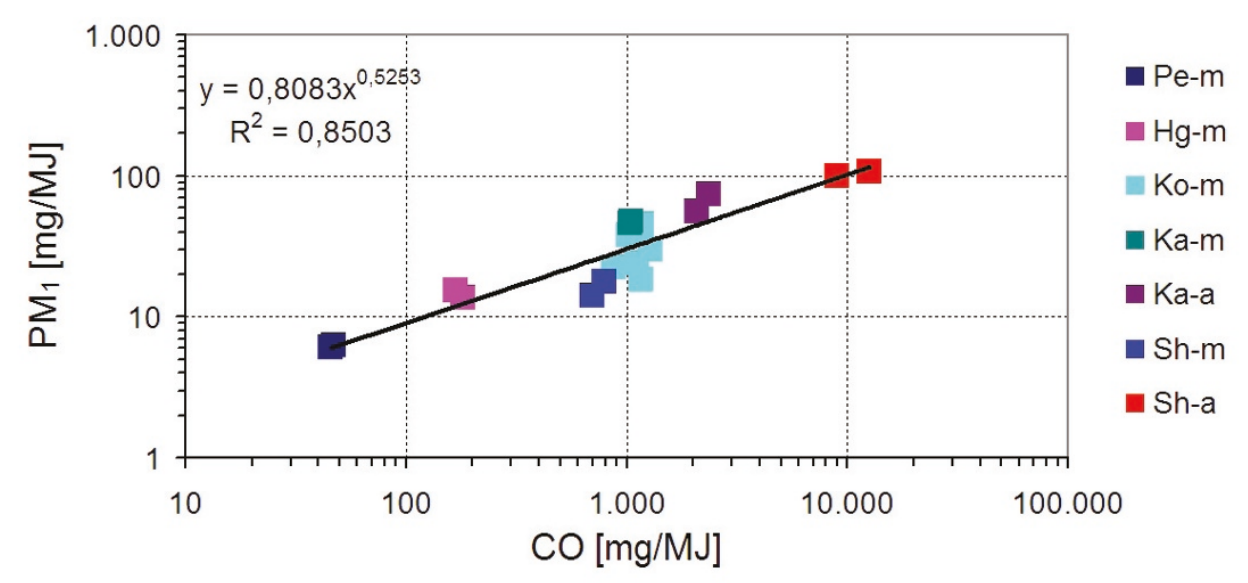

Abb. 6. Korrelation der CO- und der PM -Emissionen für alle untersuchten Biomasse-Kleinfeuerungsanlagen

Feinstaubemissionen resultieren hauptsächlich aus Fragmentierungsvorgängen während des Brennstoffabbrandes (hohe Temperaturgradienten in der Holzkohle). Die in den Feinstaubproben enthaltenen Schwermetalle ( $\mathrm{Zn}$ und $\mathrm{Pb}$ ) sowie die Elemente $\mathrm{Ca}, \mathrm{Si}$ und $\mathrm{Mg}$ wurden als Oxide berücksichtigt.

In Abb. 7 sind die mit den jeweiligen $\mathrm{PM}_{1}-$ Konzentrationen im Rauchgas gewichteten, mittleren Zusammensetzungen des anorganischen Anteils (oberes Diagramm) sowie die Gesamtzusammensetzung (anorganischer und organischer Anteil sowie Rußemissionen, unteres Diagramm) der Feinstaubemissionen zusammenfassend dargestellt. Die anorganische Fraktion der untersuchten Feinstaubproben besteht zum Großteil aus Alkalimetallverbindungen (Alkalimetallsulfate, Alkalimetallchloride und Alkalimetallkarbonate), wobei die Aufteilung innerhalb der Alkalimetallverbindungen von der S- und ClKonzentration im Brennstoff beeinflusst wird. Mit Ausnahme der Feinstaubproben aus den Testläufen mit dem modernen Kaminofen (58\%) repräsentieren die Alkalimetallverbindungen mehr als $87 \%$ der anorganischen Partikelmasse. Weiters konnte festgestellt werden, dass mit Ausnahme der Feinstaubproben des alten Stückholzkessels und des modernen Kaminofens, bei denen höhere Anteile an Alkalimetallkarbonaten und anderen Oxiden gefunden wurden, Alkalimetallsulfate die Zusammensetzung dominieren. Die verbleibende Partikelmasse besteht aus Schwermetalloxiden (hauptsächlich Zinkoxid) sowie Oxiden der Elemente $\mathrm{Ca}, \mathrm{Mg}$ und Si (andere Oxide). Durch eine ungewöhnlich hohe $\mathrm{Si}$-Konzentration in den Feinstaubproben des modernen Kaminofens (erfahrungsgemäß liegen die Si-Konzentrationen bei rund 1/5 der bei den Feinstaubproben des modernen Kaminofens gemessenen Konzentrationen) beträgt der Anteil der anderen Oxide hier rund 40 \% der anorganischen Partikelmasse. Zusätzlich zu den anorganischen Partikeln bilden sich, je nach Ausbrandqualität, Partikel aus organischem sowie elementarem Kohlenstoff 


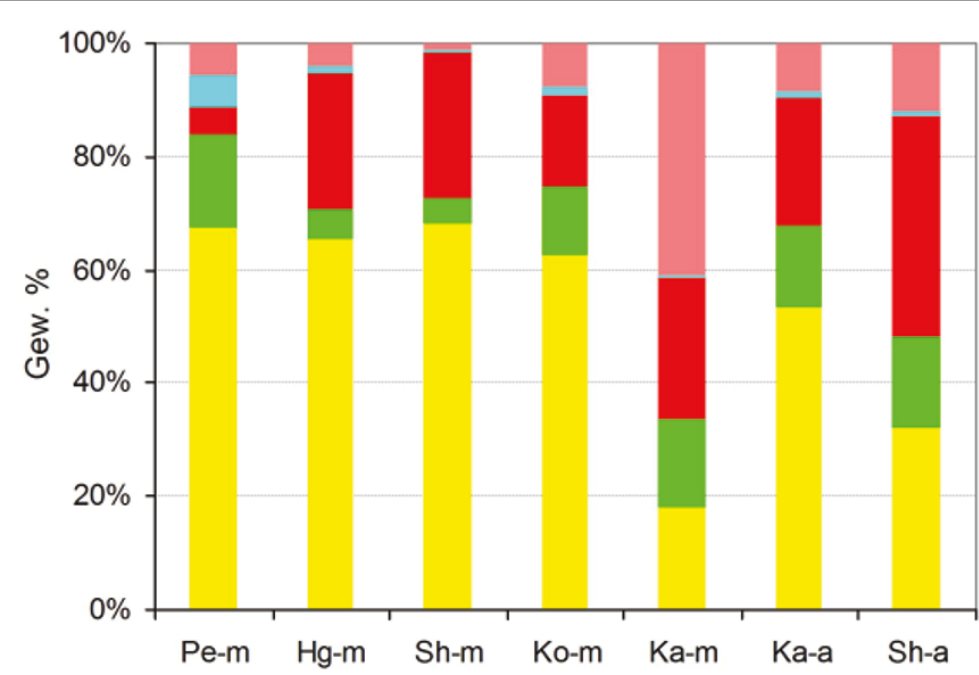

andere Oxide

- Schwermetall-Oxide

- Alkalimetall-Karbonate

- Alkalimetall-Chloride

Alkalimetall-Sulfate

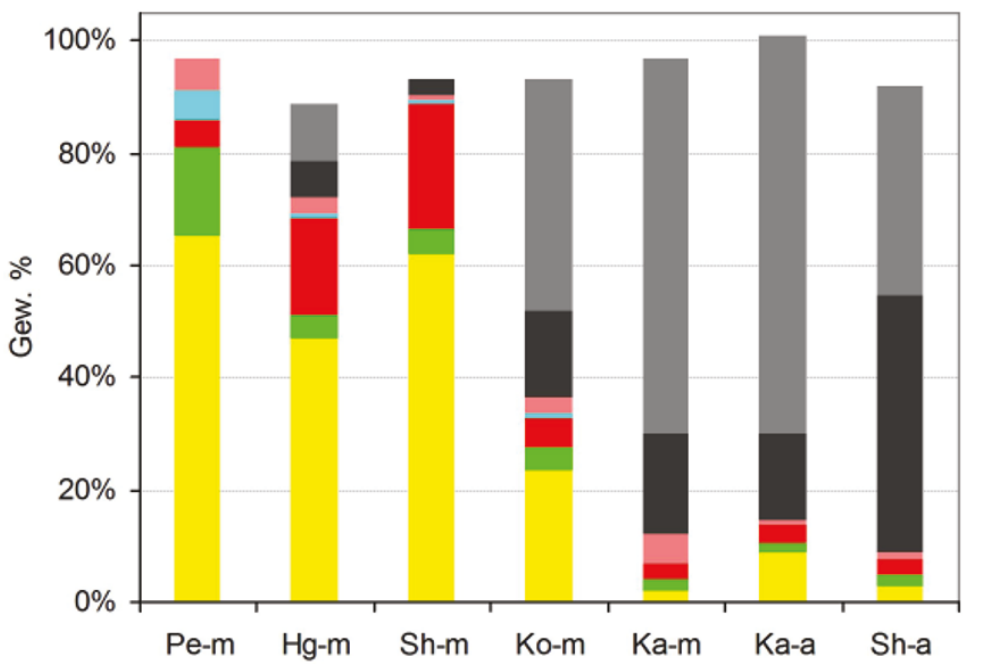

- Russ (EC)

n org. C-Verbindungen (POM)

andere Oxide

Schwermetall-Oxide

- Alkalimetall-Karbonate

Alkalimetall-Chloride

Alkalimetall-Sulfate

Abb. 7. Chemische Zusammensetzung des anorganischen Anteils (oben) sowie der Gesamtzusammensetzung (unten) der $\mathrm{PM}_{1}$-Emissionen

(EC) (Rußpartikel). Organische Kohlenstoffverbindungen enthalten zusätzlich Sauerstoff und Wasserstoff. Der Umrechnungsfaktor von der Masse an organischem Kohlenstoff (OC) auf die Masse an organischen Kohlenstoffverbindungen (insbesondere Kohlenwasserstoffe) liegt für atmosphärische Aerosole beispielsweise bei 1,2 bis 1,4 [18]. Für Aerosole aus der Holzverbrennung werden in der Literatur Faktoren von bis $\mathrm{zu} 2,0$ angegeben [19]. Die in Abb. 7 dargestellten Massen an organischen Kohlenstoffverbindungen (particulate organic matter - POM) wurden mit einem Umrechnungsfaktor von 1,4 auf Basis der OC-Analysen berechnet.

Die Feinstaubemissionen von modernen BiomasseKleinfeuerungssystemen mit erzwungener Durchströmung (moderner Pellet-, Hackgut- und Stückholzkessel) setzen sich hauptsächlich aus anorganischen Aerosolen zusammen. Die Bildung dieser anorganischen Aerosole ist vorwiegend von der Brennstoffzusammensetzung abhängig. Bei Hackgut handelt es sich im Gegensatz zu Weichholzpellets um einen deutlich inhomogeneren Brennstoff, da unterschiedliche Mischungen aus Hartholz und Weichholz mit unterschiedlichem Rindenanteil zum Einsatz kommen können. Hackgut stellt somit auch höhere Anforderungen an die eingesetzte Feuerungstechnik. Das eingesetzte Stückholz (Hartholz) hat generell einen höheren Rindenanteil und höheren Aschegehalt als Weichholz. Bei den Feinstaubproben der Testläufe mit dem Pelletkessel lagen die Konzentrationen von $\mathrm{OC}$ und $\mathrm{EC}$ unter den Bestimmungsgrenzen des Analysators. Bei Betrieb des Pelletkessels, der hauptsächlich durch Phasen vollständigen Gasphasenausbrands charakterisiert ist, werden somit nur sehr geringe 


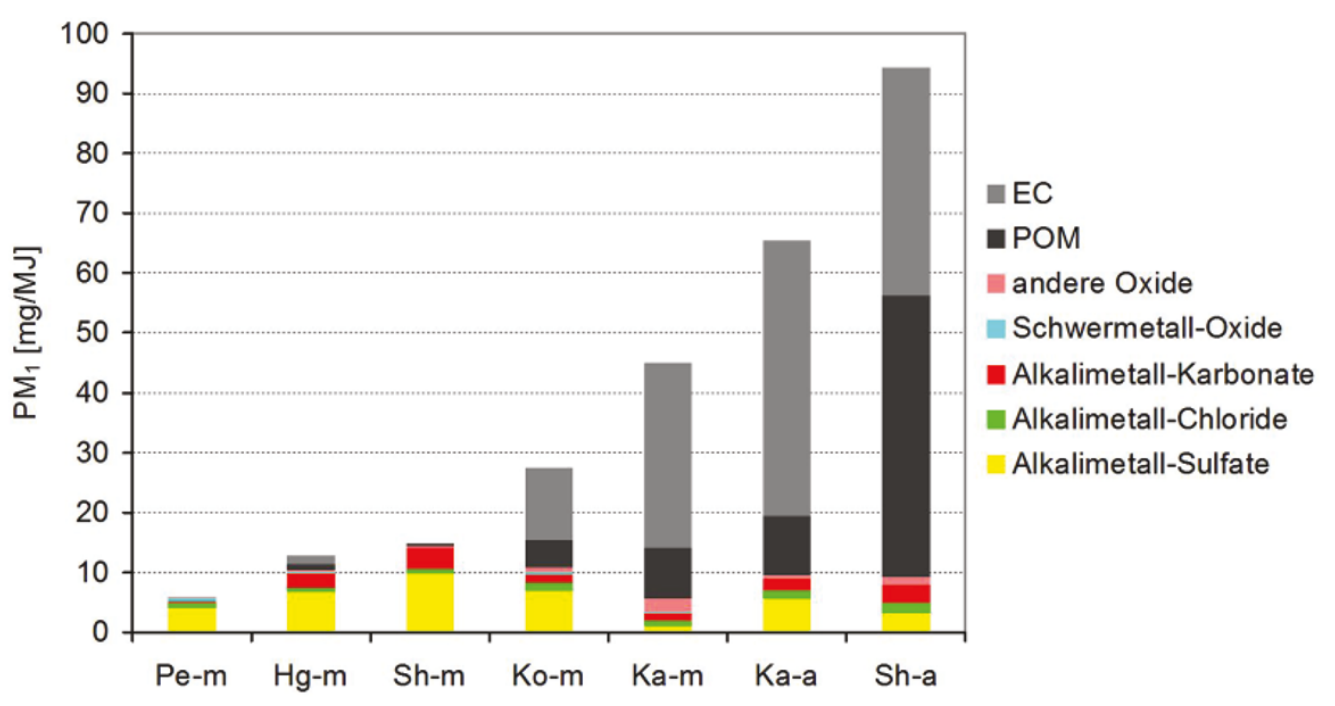

Abb. 8. $\mathrm{PM}_{1}$-Emissionen der untersuchten Biomassefeuerungen, gegliedert nach chemischen Verbindungen

Mengen an organischem Kohlenstoff und Ruß emittiert, während für die Feinstaubproben aus Testläufen mit dem Hackgutkessel leicht erhöhte Anteile von organischen Kohlenstoffverbindungen (6\%) und elementarem Kohlenstoff (10\%) festgestellt wurden. Der Großteil der organischen Fraktion bei den Feinstaubproben aus den Testläufen mit dem Hackgutkessel resultiert aus Teillastphasen, in denen die Durchmischung der Rauchgase mit der Verbrennungsluft keinen vollständigen Gasphasenausbrand gewährleistet. Für die Feinstaubproben aus den Testläufen mit dem modernen Stückholzkessel konnten nur sehr geringe Anteile organischer Kohlenstoffverbindungen (3\%) festgestellt werden, die hauptsächlich während der Anbrennphase entstehen. Bei den untersuchten Naturzugöfen (Kaminöfen und Kachelofen) sowie beim alten Stückholzkessel spielen die anorganischen Aerosole eine eher untergeordnete Rolle. Die Partikel werden hauptsächlich von organischen Kohlenstoffverbindungen und Rußemissionen dominiert. Während bei den Naturzugöfen die kohlenstoffhaltige Fraktion zum überwiegenden Teil (> 73\%) aus elementarem Kohlenstoff besteht, überwiegen organische Kohlenstoffverbindungen bei den Feinstaubproben des alten Stückholzkessels mit rund 55\% der Gesamtpartikelmasse, welche insbesondere aus unvollständigem Gasphasenausbrand resultieren. Die hohen Rußemissionen bei den Naturzugöfen lassen auf Luftmangel bei hohen Temperaturen in der Brennkammer schließen (unvollständige Durchmischung der Rauchgase mit der Verbrennungsluft) [16].

Die Ergebnisse der chemischen Analysen zeigen, dass ein Grundlevel der Feinstaubemissionen aus der Bildung anorganischer Partikel und Emissionssteigerungen aus der Bildung von organischen Partikeln sowie Rußpartikeln aufgrund schlechter werdender Ausbrandqualität resultieren (siehe Abb. 8). Es konnte festgestellt werden, dass moderne, dem aktuellen Stand der Technik entsprechende, Feuerungen mit erzwungener Durchströmung (moderner Pellet-, Hackgut- und Stückholzkessel) verglichen mit Altanlagen und händisch beschickten Naturzugöfen (Kaminöfen und Kachelofen) deutlich geringere Emissionen an organischem Kohlenstoff (OC) und Ruß (EC) aufgrund verbesserten Gasphasenausbrands aufweisen (bis zu einem Faktor 100 geringer für den Vergleich des modernen Pelletkessels mit dem alten Scheitholzkessel). Dies ist vor allem auf eine gegenüber Altanlagen und Naturzugöfen bessere Luftstufung sowie bessere Durchmischung der Rauchgase mit der Verbrennungsluft zurückzuführen. Ein weiterer Vorteil von Kesselsystemen ist der quasikontinuierliche Betrieb gegenüber dem chargenweisen Betrieb von Naturzugsystemen.

Neben den Gehalten an anorganischen Feinstaubbildnern sowie organischem und elementarem Kohlenstoff wurden auch die Gehalte an polyzyklischen aromatischen Kohlenwasserstoffen (PAKs) in den Feinstaubemissionsproben bestimmt. Es ist allgemein bekannt, dass PAKs eine gesundheitsschädigende Wirkung auf den Menschen haben können. PAKs können zum Beispiel die kanzerogene Wirkung anderer Chemikalien fördern, d.h. sie fungieren als Tumorpromotoren. Hierzu wurden die mit dem DGI gesammelten Feinstaubproben auf die Gehalte von insgesamt 30 PAKs analysiert. Mit der Auswahl der analysierten PAKs wurden sowohl die EPA-PAKs (16 PAKs, welche von der US-EPA (United States Environmental Protection Agency) als Leitsubstanzen für umweltanalytische Untersuchungen vorgeschlagen werden), die IARC PAKs (jene PAKs, welche von der IARC (International Agency 
Tabelle 3. Partikelgebundene PAK-Emissionen in $\mu \mathrm{g} / \mathrm{Nm}^{3}$

\begin{tabular}{|c|c|c|c|c|c|c|c|}
\hline Feuerung & Testlauf & $\begin{array}{l}\text { Summe } \\
\text { PAK }\end{array}$ & $\begin{array}{l}\text { Summe } \\
\text { EPA-PAK }\end{array}$ & $\begin{array}{l}\text { Summe } \\
\text { IARC }\end{array}$ & $\begin{array}{l}\text { Benzo[a]- } \\
\text { pyren }\end{array}$ & $\begin{array}{l}\text { Summe } \\
\text { WHO }\end{array}$ & $\begin{array}{c}\text { Summe } \\
\text { EU-Richtlinie }\end{array}$ \\
\hline \multirow[t]{2}{*}{ Pe-m } & 1 & 14,6 & 12,2 & 5,0 & 0,7 & 8,8 & 2,6 \\
\hline & 2 & 6,0 & 4,9 & 2,3 & 0,2 & 3,8 & 1,2 \\
\hline \multirow[t]{2}{*}{$\mathrm{Hg}-\mathrm{m}$} & 1 & 8,4 & 6,7 & 2,9 & 0,2 & 4,7 & 1,2 \\
\hline & 2 & 7,3 & 5,8 & 2,5 & 0,2 & 4,0 & 1,0 \\
\hline \multirow[t]{2}{*}{ Sh-m } & 1 & 103,9 & 89,6 & 24,2 & 3,6 & 36,9 & 12,2 \\
\hline & 2 & 104,9 & 88,0 & 30,0 & 5,2 & 44,5 & 15,8 \\
\hline \multirow[t]{2}{*}{ Ko-m } & 1 & 98,9 & 81,3 & 33,1 & 5,9 & 56,1 & 19,7 \\
\hline & 2 & 80,8 & 69,5 & 21,0 & 3,0 & 40,2 & 11,4 \\
\hline \multirow[t]{2}{*}{ Ka-m } & 1 & 465,9 & 387,9 & 143,2 & 27,2 & 262,7 & 78,6 \\
\hline & 2 & 263,0 & 219,4 & 80,8 & 13,1 & 145,7 & 41,8 \\
\hline \multirow[t]{2}{*}{ Ka-a } & 1 & 8786,3 & 7107,9 & 2966,7 & 499,1 & 3725,9 & 1522,0 \\
\hline & 2 & 4561,1 & 3585,5 & 1783,2 & 327,8 & 2402,3 & 961,1 \\
\hline \multirow[t]{2}{*}{ Sh-a } & 1 & $18.853,8$ & $16.768,0$ & $3.958,7$ & 579,2 & $6.474,3$ & $2.191,7$ \\
\hline & 2 & $3.385,1$ & $2.948,1$ & 849,3 & 120,8 & $1.413,7$ & 472,5 \\
\hline
\end{tabular}

for Research on Cancer) beim Menschen als krebserregend eingestuft werden) [20], die 13 von der WHO (World Health Organisation) [21] als genotoxisch eingestuften PAKs, sowie die in der Richtlinie 2004/107/ EG des Europäischen Parlaments und des Rates über Arsen, Cadmium, Quecksilber, Nickel und polyzyklische aromatische Kohlenwasserstoffe in der Luft definierten PAKs abgedeckt. In dieser EU-Richtlinie ist Benzo[a] pyren als Marker für das karzinogene Risiko durch PAKs in der Luft definiert und mit einem Grenzwert belegt. Um den Beitrag von Benzo[a]pyren besser beurteilen zu können, werden auch andere relevante polyzyklische aromatische Kohlenwasserstoffe, wenn auch nur begrenzt, überwacht. Diese Verbindungen umfassen Benzo[a]anthracen, Benzo[b]fluoranthen, Benzo[j] fluoranthen, Benzo[k]fluoranthen, Indeno[1,2,3-cd]pyren und Dibenz[a,h]anthracen. Die Überwachungsstellen für diese polyzyklischen aromatischen Kohlenwasserstoffe werden mit Probenahmestellen für Benzo[a]pyren zusammengelegt.

In Tabelle 3 sind die Ergebnisse der PAK-Analysen zusammengefasst. Die Ergebnisse sind in $\mu \mathrm{g} / \mathrm{Nm}^{3}$ angegeben und wurden aus den PAK-Konzentrationen der Feinstaubproben sowie den mittleren $\mathrm{PM}_{1}$-Emissionen über den jeweiligen Tageslastverlauf berechnet. Für die Testläufe mit dem Kachelofen wurden die gesammelten Feinstaubproben von jeweils 3 Testläufen $\mathrm{zu}$ einer Mischprobe zusammengefasst. Es sind sehr starke Unterschiede bezüglich der Summe der partikelgebundenen PAKs zwischen den untersuchten BiomasseKleinfeuerungen zu erkennen. Während sie im Vergleich der beiden Testläufe mit den jeweiligen Feuerungen für die modernen Feuerungstechnologien größtenteils auf einem ähnlichen Niveau liegen treten bei den ermittelten PAK-Gehalten der Testläufe mit den alten Feuerungstechnologien größere Schwankungen auf. Diese Schwankungen sind wiederum ein Indiz für instabile Prozessbedingungen, welche bei alten Feuerungstechnologien häufiger und längerfristiger auftreten als bei modernen Technologien. Die Proben aus den Testläufen am Pelletkessel und am Hackgutkessel weisen die geringsten und jene aus den Testläufen mit dem alten Stückholzkessel bzw. dem Kaminofenbilligprodukt die höchsten PAK-Gehalte auf. Weiters konnte festgestellt werden, dass bei allen untersuchten Feinstaubproben mehr als $79 \%$ der PAK-Gehalte den EPA-PAKs zuzuordnen sind. Der Anteil der IARC-PAKs schwankt zwischen 21 und 39\% und der der WHO-PAKs zwischen 34 und 63\%. Der Anteil des erwiesenermaßen krebserregenden Benzo[a]pyren liegt zwischen 2 und $7 \%$.

Innerhalb der Testläufe mit den jeweiligen BiomasseKleinfeuerungen sind die Zusammensetzungen der partikelgebundenen PAKs miteinander vergleichbar. Die am höchsten konzentrierten PAKs sind Phenantren, Fluoranthen, Pyren, Benzo[a]anthracen, Cyclopenta[c,d] pyren, Benzo[b]fluoranthen, Benzo[a]pyren und Benzo[g,h,i]perylen sowie bei den beiden Stückholzkesseln zusätzlich Fluoren. Mit diesen PAKs werden zwischen 77 und $86 \%$ der Gesamtheit der partikelgebundenen PAKs abgedeckt.

Benzo[a]pyren ist laut IARC der einzige beim Menschen erwiesenermaßen krebserregende PAK. Aus diesem Grund wurde der Zusammenhang zwischen den Benzo[a]pyren-Emissionen sowie der Gesamtheit der PAK-Emissionen untersucht. Wie Abb. 9 verdeutlicht, steigen die partikelgebundenen Benzo[a]pyren-Emissionen 


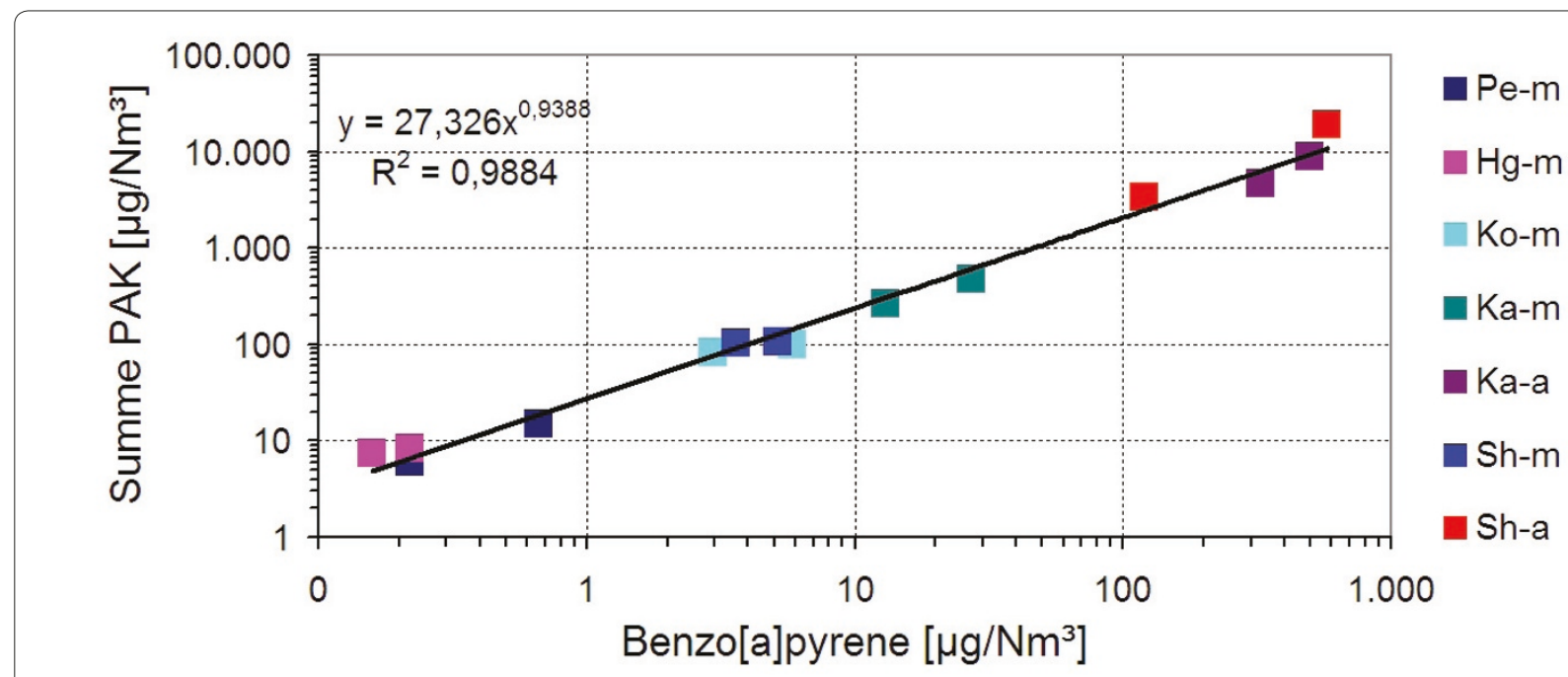

Abb. 9. Korrelation der Summe der partikelgebundenen PAK-Emissionen und der Benzo[a]pyren-Emissionen

mit steigenden Emissionen der Summe an partikelgebundenen PAKs $\left(\mathrm{R}^{2}=0,9884\right)$ und liegen statisch als Trend $(\mathrm{p}<0,1)$ vor. Wie erwartet bestehen hohe Korrelationen (Ergebnisse nicht dargestellt) zwischen den partikelgebundenen PAK-Konzentrationen und den CO- $\quad\left(R^{2}=0,8082, \quad p>0,1\right) \quad$ bzw. org.C-Emissionen $\left(\mathrm{R}^{2}=0,8647, \mathrm{p}<0,1\right)$ was wiederum bestätigt, dass die Ausbrandbedingungen einen wesentlichen Einfluss auf die Bildung von PAKs haben [22].

\section{Zusammenfassung und Schlussfolgerungen}

Die für Europa derzeit relevantesten Biomasse-Kleinfeuerungstechnologien (moderner Pelletkessel, moderner Hackgutkessel, moderner sowie alter Stückholzkessel, moderner sowie alter Kaminofen und moderner Kachelofen) wurden in umfangreichen Technikumstestläufen hinsichtlich ihrer gasförmigen und partikelförmigen Emissionen untersucht. Bei der Durchführung der Testläufe wurde speziell darauf geachtet, typische Tageslastverläufe für die jeweilige Feuerung abzufahren. Die abgefahrenen Tageslastverläufe sollten dabei den Feld- bzw. Praxisbetrieb möglichst gut abbilden. Während der Testläufe wurden die gas- und partikelförmigen Emissionen (Gesamtstaub- und Feinstaubemissionen) gemessen und aufgezeichnet sowie Partikelproben gezogen. Mit diesen Partikelproben wurden nachfolgend chemische Analysen durchgeführt.

Über den jeweiligen Tageslastverlauf konnten Feinstaubemissionen von 9,0 und $9,4 \mathrm{mg} / \mathrm{Nm}^{3}$ (bezogen auf trockenes Rauchgas und 13 Vol.\% Restsauerstoff) (6,0 und 6,2 mg/MJ) für den modernen Pelletkessel, 20,3 und $22,9 \mathrm{mg} / \mathrm{Nm}^{3}$ (13,6 und 15,3 mg/MJ) für den modernen Hackgutkessel, 21,2 und 26,4 mg/ $\mathrm{Nm}^{3}$ (14,2 und 17,6 mg/ MJ) für den modernen Stückholzkessel, 147,9 und
159,2 $\mathrm{mg} / \mathrm{Nm}^{3}$ (98,6 und 106,1 mg/MJ) für den alten Stückholzkessel, 69,2 und $70,9 \mathrm{mg} / \mathrm{Nm}^{3}$ (46,1 und 47,2 mg/MJ) für den modernen Kaminofen sowie 83,3 und $111,3 \mathrm{mg} / \mathrm{Nm}^{3}$ (55,5 und 74,2 $\mathrm{mg} / \mathrm{MJ}$ ) für das Kaminofenbilligprodukt mit veralteter Technologie ermittelt werden. Die gemessenen Feinstaubemissionen bei den Testläufen mit dem modernen Kachelofen lagen im Bereich von 27,6 bis 68,1 mg/ $\mathrm{Nm}^{3}$ (18,4 und 45,4 mg/ MJ). Bei den gasförmigen Emissionen wurden ebenfalls große Unterschiede zwischen den einzelnen Feuerungstechnologien festgestellt. Die ermittelten gasförmigen Emissionen schwanken für CO zwischen $68 \mathrm{mg} / \mathrm{Nm}^{3}$ (45 mg/MJ) für den modernen Pelletkessel bis $\mathrm{zu}$ $18.948 \mathrm{mg} / \mathrm{Nm}^{3} \quad(12.632 \mathrm{mg} / \mathrm{MJ})$ für den alten Stückholzkessel und für org.C zwischen $2,6 \mathrm{mg} / \mathrm{Nm}^{3}$ $(1,7 \mathrm{mg} / \mathrm{MJ})$ für den modernen Pelletkessel bis $\mathrm{zu}$ $1.715 \mathrm{mg} / \mathrm{Nm}^{3}$ (1.144 mg/MJ) für den alten Stückholzkessel. Für die Zusammenhänge zwischen der Bildung der gasförmigen ( $\mathrm{CO}$ und org.C) Emissionen sowie der Feinstaubemissionen wurden sehr gute Korrelationen gefunden. Zusammenfassend konnte festgestellt werden, dass moderne, dem aktuellen Stand der Technik entsprechende Biomasse-Kleinfeuerungen wesentlich geringere gasförmige und partikelförmige Emissionen aufweisen als Altanlagen bzw. Billigprodukte mit unausgereifter Verbrennungstechnologie. Weiters wurde festgestellt, dass moderne Pellet-, Hackgut- sowie Stückholzkessel wesentlich geringere gasförmige und vor allem partikelförmige Emissionen als manuell beschickte Stückholzkessel älterer Bauart und Naturzugöfen (Kaminöfen und Kachelöfen) aufweisen. Die Ausbrandqualität sinkt somit von modernen automatisch geregelten Biomasse-Kleinfeuerungen (Pellet-, Hackgutund Stückholzkessel) über moderne Naturzugöfen 
(Kaminofen und Kachelofen) hin zum Kaminofenbilligprodukt mit veralteter Technologie und dem alten Stückholzkessel.

Anhand der Ergebnisse der chemischen Analysen von Feinstaubproben konnte festgestellt werden, dass moderne Feuerungen mit erzwungener Durchströmung (Pellet-, Hackgut- und Stückholzkessel) verglichen mit Altanlagen und händisch beschickten Naturzugöfen (Kaminöfen und Kachelofen) deutlich geringere Emissionen an organischem Kohlenstoff $(\mathrm{OC})$ und Ruß (EC) aufweisen. Die OC- und EC-Emissionen resultieren aus unvollständiger Verbrennung sowie unvollständiger Oxidation von Rußpartikeln. Über den gesamten Tageslastverlauf gesehen, treten bei den modernen Feuerungen mit erzwungener Durchströmung diese Phasen nur sehr kurzzeitig auf. Bei diesen Feuerungen bestehen die Feinstaubemissionen hauptsächlich aus anorganischen Bestandteilen (Alkalimetallsulfate, Alkalimetallchloride, Alkalimetallkarbonate sowie geringen Anteilen an Schwermetalloxiden und Oxiden der Elemente $\mathrm{Ca}, \mathrm{Mg}, \mathrm{Mn}$ und $\mathrm{Si}$ ). Der alte Stückholzkessel sowie die Naturzugöfen zeigen über den Tageslastverlauf längere Phasen unvollständigen Gasphasenausbrands und somit ist es auch nachvollziehbar, dass die Feinstaubemissionen von diesen Feuerungen hauptsächlich aus OC- und EC-Emissionen bestehen. Ein wesentlicher Unterschied zwischen modernen automatischen Biomasse-Kleinfeuerungsanlagen und Altanlagen besteht darin, dass Neuanlagen mit erzwungener Durchströmung auf Grund der verbesserten und automatischen Regelung und verbesserter Durchmischung der Rauchgase mit der Verbrennungsluft auch bei instationären Lastzuständen nur geringe Mengen an organischem bzw. elementarem Kohlenstoff mit dem Feinstaub emittieren. Beim Kachelofen konnten geringere Emissionen an organischen Aerosolen und Ruß im Vergleich zu Kaminöfen festgestellt werden (wahrscheinlich aufgrund der besseren Isolierung und höheren Feuerraumtemperatur). Die Ergebnisse der Bestimmungen der Gehalte an partikelgebundenen polyzyklischen aromatischen Kohlenwasserstoffen (PAKs) bestätigen, dass die Ausbrandbedingungen einen wesentlichen Einfluss auf die Bildung von PAKs haben. Die Zusammenhänge der PAKEmissionen und der CO- und org.C-Emissionen sowie folglich der Ausbrandqualität zeigten gute Korrelationen. Schlechter werdende Ausbrandqualität führt somit zur verstärkten Bildung von PAK-Emissionen, die wiederum das Gesundheitsgefährdungspotential von Feinstaubemissionen erhöhen. Dies wird durch die hohe Korrelation der Summe der Gehalte an partikelgebundenen PAKs und der Benzo[a]pyren-Emissionen bestätigt, welche als Marker für das karzinogene Risiko durch PAKs in der Luft definiert sind und das einzige beim Menschen erwiesenermaßen krebserregende PAK darstellen. Die am höchsten konzentrierten partikelgebundenen PAKs sind Phenantren, Fluoranthen, Pyren, Benzo[a]anthracen, Cyclopenta[c,d]pyren, Benzo[b] fluoranthen, Benzo[a]pyren und Benzo[g,h,i]perylen sowie bei den Stückholzkesseln zusätzlich Fluoren.

Trotz der vergleichsweise geringen Feinstaubemissionen der untersuchten modernen Biomasse-Kleinfeuerungen sind Optimierungspotentiale nach wie vor sowohl bei den automatischen Anlagen (optimierte Feuerungskonzepte und verbesserte Anlagenregelung) und insbesondere bei Naturzugöfen (optimiertes Ofendesign, bessere Luftstufung, automatische Regelung) vorhanden. Bei Biomasse-Kleinfeuerungen, die über automatische Regelungen verfügen, ist es von besonderer Bedeutung, dass das Anlagenkonzept und das Regelungskonzept eine optimale Luftstufung und möglichst hohe Feuerraumtemperaturen gewährleisten. Wesentlich ist dabei, dass die Regelung speziell bei Anfahrvorgängen und Lastwechseln rasch auf die geänderten Randbedingungen anspricht und das Anlagenkonzept so gestaltet ist, dass auch bei Teillast ausreichend hohe Feuerraumtemperaturen vorherrschen. Maßnahmen im Zuge der Konzeption des Heizungssystems, die das Auftreten rascher bzw. häufiger Lastwechsel vermeiden helfen (z.B. Einsatz eines Pufferspeichers), wirken sich prinzipiell positiv auf den Feuerungsbetrieb und die dabei auftretenden Emissionen auf. Stückholzfeuerungen sollten prinzipiell nie ohne Pufferspeicher betrieben werden. Bei Naturzugöfen erfolgt die Verbrennungsluftzufuhr (wird im Allgemeinen als Primär-, Sekundärund Scheibenluft eingebracht) als auch der Abtransport der Rauchgase über den Kaminzug. Es werden somit keine elektrisch angetriebenen Förderaggregate (Ventilatoren) eingesetzt. Neben der Optimierung der Brennkammer bzw. des Ofendesigns und einer verbesserten Luftstufung bieten automatische Regelungskonzepte wesentliche Optimierungspotentiale hinsichtlich der Reduktion gas- und partikelförmiger Emissionen bei Naturzugsystemen [23]. Durch automatische Regelungskonzepte wird sichergestellt, dass über den gesamten Abbrand eine bestmögliche Durchmischung der Rauchgase mit der Verbrennungsluft und somit ein nahezu vollständiger Gasphasenausbrand gewährleistet wird. Weiter tragen automatische Regelungen dazu bei, Benutzerfehler, die den Abbrand ebenfalls negativ beeinflussen, $\mathrm{zu}$ minimieren bzw. fast gänzlich $\mathrm{zu}$ vermeiden.

Maßnahmen zur Minderung der organischen Aerosolemissionen und Ruß sind immer in Zusammenhang mit der Verbesserung der Ausbrandqualität zu sehen, und wirken sich dementsprechend in zweifacher Hinsicht, nämlich auf die Minderung gasförmiger ( $\mathrm{CO}$ und org.C) wie auch auf die Minderung von gesundheitsgefährdenden partikelförmigen Emissionen, aus. Speziell bei 
händisch beschickten Feuerungen (Stückholzkessel, Naturzugöfen) können Nutzerfehler einen wesentlichen Einfluss auf die Höhe der Emissionen haben. Diesbezüglich wäre es notwendig in den Bedienungsanleitungen die richtige Betriebsweise darzustellen bzw. Schulungen anzubieten.

Es wird hinsichtlich der ermittelten Feinstaubemissionen dringend empfohlen, den Austausch von Altanlagen durch moderne Biomasse-Kleinfeuerungen $\mathrm{zu}$ forcieren und entsprechend zu fördern, um einerseits die Feinstaubemissionen zu reduzieren und andererseits die Emissionen von gesundheitsgefährdenden kohlenstoffhaltigen Aerosolen zu minimieren. Würde man alle mit Holz betriebenen Altanlagen in Österreich durch moderne Biomasse-Kleinfeuerungen ersetzen, könnten rund $75 \%$ der Feinstaubemissionen, die durch Holzheizungen derzeit verursacht werden, vermieden werden. Eine Nachrüstung von Altanlagen mit Filtern, wie sie auch immer wieder angeregt wird, ist derzeit nicht sinnvoll, da, wie internationale Studien belegen, noch keine entsprechenden Filtersysteme, die auch für einen effizienten Langzeitbetrieb in Altanlagen geeignet sind, am Markt verfügbar sind. Diesbezüglich sind die Entwicklungen der nächsten Jahre noch abzuwarten.

Mit der Durchführung der in diesem Artikel beschriebenen Arbeiten stehen umfangreiche, verwertbare Daten von Testläufen mit modernen und alten BiomasseKleinfeuerungen über einen praxisnahen Tageslastverlauf zur Verfügung, die die Zusammenhänge zwischen Feuerungstechnologie sowie partikelförmigen und gasförmigen Emissionen nachvollziehbar darstellen und auch eine wichtige Basis für die Berechnung von Emissionsfaktoren bilden.

\section{Abkürzungen}

Pe-m: moderner Pelletkessel; Hg-m: moderner Hackgutkessel; Sh-m: moderner Stückholzkessel; Sh-a: alter Stückholzkessel; Ka-m: moderner Kaminofen, Ka-a: Kaminofenbilligprodukt mit veralteter Technologie; Ko-m: moderner Kachelofen; FS: Frischsubstanz; TS: Trockensubstanz

\section{Konkurrierende Interessen}

Die Autoren erklären, dass sie keine konkurrierenden Interessen haben.

\section{Beiträge der Autoren}

JK: Ausarbeitung und Konzeption des Manuskripts, TB: Einarbeitung grundlagenorientierter Aspekte, IO: Revision des Textes. Alle Autoren haben das Manuskript gelesen, geprüft und ihr Einverständnis erklärt.

\section{Danksagung}

Die in dieser Veröffentlichung beschriebene Arbeit ist im Rahmen eines Projekts der BIOENERGY 2020+ GmbH (Kplus-Programm) entstanden, welches mit Mitteln der Republik Österreich sowie der Länder Steiermark und Niederösterreich gefördert wurde.

\section{Author details}

'BIOENERGY 2020+ GmbH, Inffeldgasse 21b, A-8010 Graz, Österreich. 2Institut für Prozess- und Partikeltechnik, Technische Universität Graz, Inffeldgasse 21a, A-8010 Graz, Österreich. ${ }^{3}$ BIOS BIOENERGIESYSTEME GmbH, Inffeldgasse 21b, A-8010 Graz, Österreich.

Published: 8 March 2012
Literatur

1. Gaegauf CK, Schmid MR, Güntert P: Elemental and organic carbon in flue gas particles of various wood combustion systems. In Proceedings 8th International Conference on Energy for a Clean Environment, Lisbon; 2005

2. Hartmann H, Ellner-Schuberth F, Turowski P, Lenz V, Gerth J: Quantification and Characterisation of Particle Emissions from Residential Wood Stoves and Boilers. In: Proceedings 16th European Biomass Conference \& Exhibition - from Research to Industry and Markets, Conference in Valencia, Spain, 2-6 June 2008. ETA-Florence Renewable Energies, Italy; 2006: 1451-1457

3. Johansson LS, Leckner B, Gustavsson L, Cooper D, Tullin C, Potter A: Emission characteristics of modern and old-type residential boilers fired with wood logs and wood pellets. Atmospheric Environment 2004, 38:4183-4195

4. Johansson LS, Leckner B, Gustavsson L, Cooper D, Potter A, Tullin C, Nerntsen M: Particle emissions from residential biofuel boilers and stoves - old and modern techniques. In Proceedings of the International Seminar "Aerosols in Biomass Combustion", March 2005, Graz, Austria, book series "Thermal Biomass Utilization", Volume 6, ISBN 3-9501980-2-4, BIOS BIOENERGIESYSTEME GmbH, Graz, Austria (ed.); 2005: 145-150

5. Jokiniemi J et al.: Biomass combustion in residential heating: particulate measurements, sampling and physicochemical and toxicological characterisation. Final report of the ERA-NET Bioenergy Project "Biomass-PM", University of Kuopio, ISSN 0786-4728, Kuopio, Finland; 2008

6. Obernberger I, Brunner T, Bärnthaler G: Aktuelle Erkenntnisse im Bereich der Feinstaubemissionen bei Pelletfeuerungen. In: Tagungsband zum 5. Industrieforum Holzenergie. Stuttgart: Deutscher Energie-Pellet-Verband e.v. and Deutsche Gesellschaft für Sonnenenergie e.V. (ed.); 2005: 54-64

7. Wieser U, Gaegauf CK, Macquat Y: Feldmesskampagne Partikelemissionen aus Holzfeuerungen - Untersuchung der Partikelfrachten in Holzfeuerungsabgasen unter Praxisbedingungen. Ökozentrum Langenbruck; 2001

8. Klippel N, Nussbaumer T: Feinstaubbildung in Holzfeuerungen und Gesundheitsrelevanz im Vergleich zu Dieselruss. In: Feinstaubminderung und Stromerzeugung im Rahmen der zukünftigen Energieversorgung. 9. Holzenergie-Symposium, Tagung an der ETH Zürich am 20.10.2006, Verenum Zürich

9. Wilson WE, Suh HH: Fine particles and coarse particles: concentration relationship relevant to epidemiological studies. J. Air \&Waste Management Association 1997, 47:1238-1249

10. Brunner T: Aerosols and coarse fly ashes in fixed-bed biomass combustion Graz University of Technology, PhD-thesis, book series "Thermal Biomass Utilization", Volume 7, ISBN 3-9501980-2-4

11. Jungmeier G, Golja F, Spitzer J: Der technologische Fortschritt bei Holzfeuerungen - Ergebnisse einer statistischen Analyse der Prüfstandsmessungen der BLT Wieselburg von 1980 bis 1998. Schriftenreihe des BMUJF, Band 11/1999, ISBN 3-901 271-98-8, Graz, Austria; 1999

12. Spitzer J, Enzinger P, Fankhauser G, Fritz W, Golja F, Stiglbrunner R: Emissionsfaktoren für feste Brennstoffe. Final report, Institute of Energy Research, Joanneum Research, Graz, Austria; 1998

13. Bäfver $L$ : Particles from biomass combustion - Characteristics and influence of additives. PhD-thesis, Chalmers University of Technology; 2008

14. Boman C: Particulate and gaseous emissions from residential biomass combustion. PhD-thesis, Umeå University; 2005

15. Sippula O, Hokkinen J, Lamberg H, Puustinen H, Yli-Pirilä P, Tissari J, Jokiniemi $\mathrm{J}$ : Particle emissions from small biomass and fuel oil fired heating units. In: Proceedings of the 17th European Biomass Conference \& Exhibition, June 2009, Hamburg, ETA-Renewable Energies (Ed.), Italy; 2009

16. Wiinikka $\mathrm{H}$ : High temperature aerosol formation and emission minimisation during combustion of wood pellets. PhD-thesis, Luleå University of Technology; 2005

17. Wiinikka $\mathrm{H}, \mathrm{Gebart} \mathrm{R}$ : The influence of air distribution rate on particle emissions in fixed bed combustion of biomass. Combustion Science and Technology 2005, 177:1747-1766

18. Gray HA, Cass GR, Hunzicker JJ, Heyerdahl EK, Rau JA: Characteristics of atmospheric organic and elemental carbon particle concentrations in Los Angeles. Environmental Science and Technology 1986, 20:580-589

19. Turpin JT, Lim H: Species contributions to PM25 mass concentrations: revisiting common assumptions for estimating organic mass. Aerosol Science and Technology 2001, 35: 602-610

20. International Agency for Research on Cancer: Air pollution, part 1, Some non-heterocyclic aromatic hydrocarbons and some related industrial 
exposures. Volume 92 in preparation, meeting report; 2008

21. WHO: Selected non-heterocyclic polycyclic aromatic hydrocarbons. Environmental health criteria 202. WHO International Program of Chemical Safety, Geneva Switzerland; 1998

22. Kelz J, Brunner T, Obernberger I, Jalava P, Hirvonen M.-R.: PM emissions from old and modern biomass combustion systems and their health effects. In: Proceedings of the 18th European Biomass Conference, May 2010, Lyon, France, ETA-Renewable Energies (Ed.), Italy; 2010

23. Brunner T, Obernberger I, Scharler R: Primary measures for low-emission residential wood combustion - comparison of old with optimised modern systems. In: Proceedings of the 17th European Biomass Conference \&

Exhibition, June 2009, Hamburg, ETA-Renewable Energies (Ed.), Italy; 2009

doi:10.1186/2190-4715-24-11

Cite this article as: Kelz J, et al.: Emission factors and chemical

characterisation of fine particulate emissions from modern and old

residential biomass heating systems determined for typical load cycles.

Environmental Sciences Europe 2012, 24:11. 